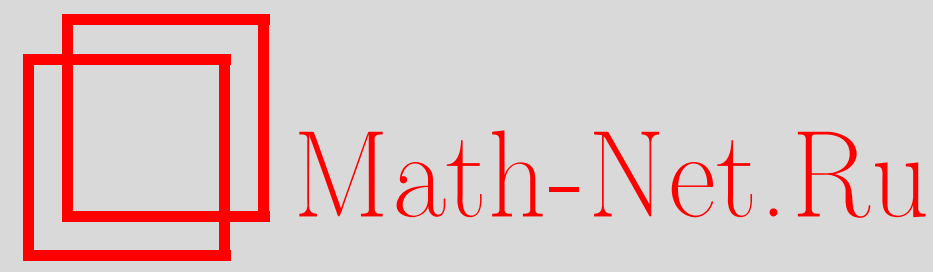

В. Д. Седых, О топологии устойчивых особенностей коранга 1 на крае связной компоненты дополнения к фронту, Матем. сб., 2004, том 195, номер 8, 91-130

DOI: https://doi.org/10.4213/sm840

Использование Общероссийского математического портала Math-Net.Ru подразумевает, что вы прочитали и согласны с пользовательским соглашением http: //www.mathnet.ru/rus/agreement

Параметры загрузки:

IP : 54.164 .48 .24

26 апреля 2023 г., 16:20:04 
УДК 515.16

\author{
В. Д. Седых
}

\title{
О топологии устойчивых особенностей коранга 1 на крае связной компоненты дополнения к фронту
}

\begin{abstract}
В работе изучаются ограничения на расположение особенностей на границе связной компоненты дополнения к волновому фронту. Предполагается, что граница компоненты компактна, является краем многообразия, а фронт имеет в точках этого края только устойчивые особенности коранга 1. При этих предположениях указьваются линейные соотношения между эйлеровьпи характеристиками многообразий особенностей на крае данной компоненты. В частности, найдены все универсалњные линейные соотношения между эйлеровьми характеристиками многообразий особенностей на краях эллиптических и гиперболических связных компонент дополнения к фронту.

Библиография 15 названий.
\end{abstract}

\section{Введение}

Рассмотрим волновой фронт обшего положения в гладком (класса $C^{\infty}$ ) многообразии (см. [1]). Это особая гиперповерхность, стратифицированная по классам особых точек. Особенности фронта удовлетворяют различньм условиям сосуществования (см., например, [2], [3]). Недавно (см. [4]) были обнаружены новые условия такого рода для фронтов, имеющих только устойчивые особенности коранга 1.

Настоящая работа развивает это направление. Теперь мы изучаем ограничения на расположение особенностей на границе связной компоненты дополнения к фронту. Предполагается, что граница компоненты компактна, является краем многообразия (а именно, краем замыкания компоненты), а фронт имеет в точках этого края только устойчивые особенности коранга 1. При этих предположениях мы указываем линейные соотношения между эйлеровыми характеристиками многообразий особенностей на крае данной компоненты.

В частности, найдены все универсальные линейные соотношения между эйлеровыми характеристиками многообразий особенностей на краях эллиптических и гиперболических связных компонент дополнения к фронту (компонента эллиптическая, если особенности фронта во всех точках ее края имеют только нечетные кратности; гиперболичность определена ниже). Они имеют следующий вид: эйлерова характеристика каждого нечетномерного многообразия особенностей на крае компоненты является линейной комбинацией эйлеровых характеристик четномерных многообразий особенностей бо́льшей коразмерности на крае той же компоненты.

Работа вьполнена при поддержке Российского фонда фундаментальных исследований (грант № 02-01-00655).

(C) В. Д. Седых 2004 
Универсальность этих комбинаций означает, что их коэффициенты зависят только от классов соответствующих особенностей и типа компоненты (эллиптическая или гиперболическая) и не зависят от того, какой фронт и в каком пространстве мы рассматриваем, какую эллиптическую (гиперболическую) компоненту его дополнения мы взяли. Других универсальных линейных соотношений между эйлеровыми характеристиками указанных многообразий не существует.

Мы описываем простой комбинаторный алгоритм для вычисления всех этих соотношений. Он основан на конструкции [4], которая позволяет строить разрешения различных циклов особенностей фронта, имеющего лишь устойчивые особенности коранга 1.

А именно, мы сопоставляем любой особенности $\mathscr{A}$ фронта гладкое многообразие $\Phi_{\mathscr{A}}$ и гладкое конечное собственное отображение $\varphi_{\mathscr{A}}$ этого многообразия в пространство, объемлющее рассматриваемый фронт, такие, что

1) образом отображения $\varphi_{\mathscr{A}}$ является замыкание многообразия особенностей $\mathscr{A}$ фронта в этом пространстве;

2) если особенность $X$ примыкает к особенности $\mathscr{A}$, то отображение $\varphi_{\mathscr{A}}$ является конечным накрытием над многообразием особенностей $X$ исходного фронта;

3) кратность этого накрытия зависит только от классов особенностей $\mathscr{A}, X$ и легко вычисляется рекуррентно.

Для $\mathscr{A}=A_{1}$ (неособые точки фронта) пара $\left(\Phi_{\mathscr{A}}, \varphi_{\mathscr{A}}\right)$ является, по сути дела, обычной нормализацией бифуркационной диаграммы особенностей коранга 1 в терминах зеркал груп Вейля $A_{\mu}$. В общем же случае кратность отображения $\varphi_{\mathscr{A}}$ над открытой частью цикла особенностей $\mathscr{A}$ может быть больше 1.

В настоящей статье указываются новые свойства конструкции [4]. В частности, точки многообразия $\Phi_{\mathscr{A}}$, которые переходят при отображении $\varphi_{\mathscr{A}}$ в особенности фронта, имеющие большую, чем у $\mathscr{A}$, коразмерность, образуют особую гиперповерхность. Мы получаем нормальные формы особенностей этой гиперповерхности относительно диффеоморфизмов многообразия $\Phi_{\mathscr{A}}$. Эти особенности устойчивы (относительно малых деформаций исходного фронта) и просты (не имеют модулей). Они приводимы и связаны с краевыми особенностями функций.

Часть полученных результатов (в эллиптическом случае) анонсирована в [5]. Автор благодарен В.И. Арнольду и М.Э. Казаряну за полезные обсуждения.

\section{§1. Соотношения между эйлеровыми характеристиками}

Пусть $\mathscr{F}$ - фронт обшего положения в гладком $n$-мерном многообразии $V$. Это особая гиперповерхность, являюшаяся образом (собственного) лежандрова отображения гладкого $(n-1)$-мерного многообразия (основные определения и факты теории особенностей лежандровых отображений см. в [1]).

Лежандрово отображение называется устойчивым лежандровым отображсением коранга 1, если оно (лежандрово) устойчиво и размерность ядра его 1-го дифференциала в каждой точке не превосходит 1. Фронт устойчивого лежандрова отображения коранга 1 назьвается устойчивым фронтом коранга 1 . Фронт лежандрова отображения связного многообразия назьвается неприводимылм. 
Особенности устойчивых фронтов коранга 1, как и (мульти)особенности соответствующих лежандровых отображений, нумеруются элементами свободной абелевой полугруппы по сложению с образующими $A_{1}, A_{2}, \ldots$ Особенности $A_{\mu_{1}}+$ $\cdots+A_{\mu_{m}}$ фронта $\mathscr{F}$ определяются следующим образом.

Рассмотрим произвольное целое положительное $\mu \leqslant n$. Через $\Delta_{\mu}(n)$ обозначим гиперповерхность в $\mathbb{R}^{n}=\left\{\left(\lambda_{0}, \ldots, \lambda_{n-1}\right)\right\}$, образованную точками, для которых многочлен

$$
t^{\mu+1}+\lambda_{\mu-1} t^{\mu-1}+\cdots+\lambda_{1} t+\lambda_{0}
$$

от $t$ имеет кратный вещественный корень.

ОПРЕДЕЛЕНИЕ 1.1. Фронт $\mathscr{F}$ имеет особенность $A_{\mu}$ в точке $x \in \mathscr{F}$, если его росток $(\mathscr{F}, x)$ в этой точке диффеоморфен ростку в нуле гиперповерхности $\Delta_{\mu}(n)$ в $\mathbb{R}^{n}$.

Точки фронта $\mathscr{F}$, в которых он имеет особенность $A_{\mu}$, образуют гладкое подмногообразие $\left(A_{\mu}\right)$ F коразмерности $\mu$ в $V$. Это многообразие, вообше говоря, незамкнуто.

ОПРЕДЕЛЕНИЕ 1.2. Фронт $\mathscr{F}$ имеет особенность $A_{\mu_{1}}+\cdots+A_{\mu_{m}}$ в точке $x$, если росток $(\mathscr{F}, x)$ состоит из $m$ неприводимых компонент $\mathscr{F}_{1}, \ldots, \mathscr{F}_{m}$ с особенностями $A_{\mu_{1}}, \ldots, A_{\mu_{m}}$ в точке $x$, причем ростки многообразий $\left(A_{\mu_{1}}\right)_{\mathscr{F}_{1}}, \ldots,\left(A_{\mu_{m}}\right)_{\mathscr{F}}$ пересекаются в этой точке трансверсально.

Ненулевые элементы указанной выше полугруппы называются классами особенностей. Нуль ฤ полугруппы мы будем использовать для обозначения дополнения $V \backslash \mathscr{F}$ к фрронту $\mathscr{F}$.

ПримеР 1.3. Фронт обшего положения в трехмерном пространстве может иметь лишь следующие особенности: $A_{1}$ (неособая точка), $2 A_{1}$ (трансверсальное пересечение двух гладких ветвей фронта), $A_{2}$ (ребро возврата), $3 A_{1}$ (трансверсальное пересечение трех гладких ветвей фронта), $A_{2}+A_{1}$ (трансверсальное пересечение ребра возврата с гладкой ветвью фронта) и $A_{3}$ (ласточкин хвост).

Особенности $A_{\mu_{1}}+\cdots+A_{\mu_{m}}$ любого фронта общего положения называются его устойчивыми особенностями коранга 1 . Числа $\mu_{1}, \ldots, \mu_{m}$ называются кратностями особенности $\mathscr{A}=A_{\mu_{1}}+\cdots+A_{\mu_{m}}$. Их сумма codim $\mathscr{A}=\mu_{1}+\cdots+\mu_{m}$ называется коразмерностью этой особенности. Число $\operatorname{deg} \mathscr{A}=\operatorname{codim} \mathscr{A}+m$ называется степенью особенности $\mathscr{A}$.

Коразмерность особенности не превьшает размерности $n$ объемлющего многообразия $V$. Множество $\mathscr{A}_{\mathscr{F}}$ особенностей $\mathscr{A}$ фронта $\mathscr{F}$ с данным (неупорядоченным) набором кратностей (т.е. множество точек фронта, в которых он имеет данную особенность) является гладким подмногообразием в $V$, коразмерность которого равна коразмерности особенности.

Пусть $\overline{\mathscr{A}}_{\mathscr{F}}$ - замыкание многообразия $\mathscr{A}_{\mathscr{F}}$ в многообразии $V$. Если множество

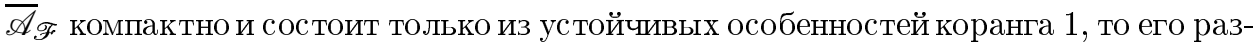
биение на связные компоненты многообразий этих особенностей является конечной $C^{\infty}$-стратификацией Уитни. Легко видеть, что эта стратификация минимальна среди всех $C^{\infty}$-стратификаций Уитни множества $\overline{\mathscr{A}}_{\mathscr{F}}$ (определение стратификации Уитни и ее свойства см., например, в [6]). 
Рассмотрим связную компоненту $U$ дополнения $V \backslash \mathscr{F}$ к фронту $\mathscr{F}$. Компонента $U$ называется правильной, если ее замыкание $\bar{U}$ в $V$ является многообразием с краем $\bar{U} \backslash U$ (т.е. подмножество $\bar{U} \subset V$ в окрестности любой точки из $\bar{U} \backslash U$ гомеоморфно замкнутому полупространству в $\mathbb{R}^{n}$ ). Край замыкания правильной связной компоненты назьвается краем этой компоненты.

Замечание 1.4. Дополнение к фронту может не иметь правильных связных компонент. Например, такой фронт дает стандартная иммерсия бутылки Клейна в $\mathbb{R}^{3}$ (все особенности этой поверхности исчерпьваются двойными трансверсальньпи самопересечениями $2 A_{1}$ вдоль гладко вложенной окружности).

Рассмотрим, в частности, связные компоненты дополнения $\mathbb{R}^{n} \backslash \Delta_{\mu}(n)$. Компонента, соответствующая многочленам (1), которые имеют $\mu+1$ попарно различных вещественных корней, называется гиперболической. Компонента, соответствующая многочленам с минимально возможным числом вещественных корней (т.е. с одним вещественным корнем, если $\mu$ четное, и без таковых, если $\mu$ нечетное), называется обобщенно-әллиптической (или просто әллиптической в случае нечетного $\mu$ ).

Следующее утверждение хорошо известно.

ПРЕДЛОЖЕНИЕ 1.5. Дополнение $\mathbb{R}^{n} \backslash \Delta_{\mu}(n)$ имеет ровно две правильные связные компоненты, а именно гиперболическую и обобщенно-эллиптическую.

ДоказАТЕльство. Связные компоненты $U_{k}$ дополнения $\mathbb{R}^{n} \backslash \Delta_{\mu}(n)$ нумеруются числом $k$ вещественных корней многочлена (1). Если $1<k<\mu$, то граница компоненты $U_{k}$ содержит многочлен $P(t)$, который имеет $k-2$ простых вешественных корня и два различных вещественных корня $t_{1}, t_{2}$ кратности 2 . В любой достаточно малой окрестности $O(P)$ этого многочлена (в пространстве многочленов вида (1)) найдутся многочлены $P_{1}(t)$ и $P_{2}(t)$ такие, что

$P_{1}\left(t_{1}\right) P^{\prime \prime}\left(t_{1}\right)<0, \quad P_{1}\left(t_{2}\right) P^{\prime \prime}\left(t_{2}\right)>0, \quad P_{2}\left(t_{1}\right) P^{\prime \prime}\left(t_{1}\right)>0, \quad P_{2}\left(t_{2}\right) P^{\prime \prime}\left(t_{2}\right)<0$.

Многочлены $P_{1}, P_{2}$ лежат в компоненте $U_{k}$, но их нельзя соединить непрерывной кривой в разности $O(P) \backslash \Delta_{\mu}(n)$. Предложение 1.5 доказано.

Зафиксируем произвольную правильную связную компоненту $U$ дополнения $V \backslash \mathscr{F}$ к фронту $\mathscr{F}$. Край этой компоненты обозначим через Г. Особенности фронта $\mathscr{F}$ в точках гиперповерхности $Г$ называются особенностями самой гиперповерхности Г.

Пусть гиперповерхность Г имеет только устойчивые особенности коранга 1.

ОПРЕДЕЛЕНИЕ 1.6. Предположим, что Г имеет особенность $A_{\mu}$ в точке $x$. Тогда компонента $U$ назьвается гиперболической (обобщенно-эллиптической, эллиптической) в точке $x$, если росток в $x$ пары в $V$, образованной фронтом $\mathscr{F}$

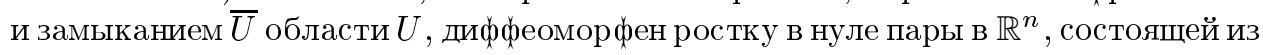
фронта $\Delta_{\mu}(n)$ и замыкания гиперболической (обобщенно-эллиптической, эллиптической соответственно) связной компоненты дополнения $\mathbb{R}^{n} \backslash \Delta_{\mu}(n)$.

ЗАмЕчАНИЕ 1.7 . Если $\mu=1$, то компонента $U$ является одновременно гиперболической и эллиптической в точке $x$. 
Компонента $U$ называется гиперболической (обобщенно-эллиптической, әллиптической), если каждая точка $x \in \Gamma$ обладает окрестностью $O(x) \subset V$, в которой множество $U \cap O(x)$ является пересечением гиперболических (обобщенно-эллиптических, эллиптических соответственно) связных компонент дополнения в $O(x)$ к неприводимым компонентам ростка $(\mathscr{F}, x)$. Нас будут интересовать в основном только гиперболические и эллиптические связные компоненты дополнения $V \backslash \mathscr{F}$.

ЗАмЕчАНИЕ 1.8. Компонента $U$ является эллиптической тогда и только тогда, когда ее край Г имеет особенности только с нечетными кратностями.

Рассмотрим множество $\mathscr{A}_{\Gamma}=\mathscr{A}_{\mathscr{F}} \cap$ Г особенностей $\mathscr{A}=A_{\mu_{1}}+\cdots+A_{\mu_{m}}$ гиперповерхности $Г$. Оно является гладким подмногообразием в многообразии $V$. Связные компоненты этого многообразия являются связными компонентами многообразия $\mathscr{A}_{\mathscr{F}}$.

Обозначим через $\chi\left(\mathscr{A}_{\Gamma}\right)$ эйлерову характеристику многообразия $\mathscr{A}_{\Gamma}$ (альтернированная сумма чисел Бетти групп гомологий с компактными носителями). Иногда, чтобы упростить громоздкие обозначения, мы будем опускать индекс Г и писать $\chi(\mathscr{A})$.

ТеОрема 1.9. Пусть Г - край правильной связной компоненты U дополнения к фронту Ғ общего положения в гладком $n$-мерном многообразии $V$. Предположим, что гиперповерхность Г компактна, фронт Я્F имеет на Г только устойчивые особенности коранга 1 , а компонента $U$ является либо гиперболической, либо эллиптической. Тогда справедливы следующие утверәсдения.

1) Для любого $\mathscr{A}=A_{\mu_{1}}+\cdots+A_{\mu_{m}}$ такого, что многообразие $\mathscr{A}_{\Gamma}$ особенностей $\mathscr{A}$ zиперповерхности $Г$ имеет нечетную размерность, эйлерова характеристика $\chi\left(\mathscr{A}_{\Gamma}\right)$ многообразия $\mathscr{A}_{\Gamma}$ является линейной комбинацией

$$
\chi\left(\mathscr{A}_{\Gamma}\right)=\sum_{X} K_{\mathscr{A}}(X) \chi\left(X_{\Gamma}\right)
$$

эйлеровых характеристик четномерных многообразий $X_{\Gamma}$ особенностей $X$ гиперповерхности $\Gamma$, әде $\operatorname{codim} X>\operatorname{codim} \mathscr{A}$. Эта комбинация универсальна в том смысле, что каждый ее коэффициент $K_{\mathscr{A}}(X)$ зависит только от классов особенностей $\mathscr{A}, X$ и типа компоненты $U$ (әиперболическая или эллиптическая) $и$ не зависит от $n, V$, F $и$ выбора компоненты $U$ среди правильных связных компонент дополнения $V \backslash \mathscr{F}$ данного типа.

2) Список формул (2) для особенностей $\mathscr{A}$ коразмерности $c \leqslant 6$ в случае гиперболической компоненты $U$ приведен в таблице 1 для нечетного $n$ (многоточие обозначает линейную комбиначию эйлеровых характеристик многообразий особенностей нечетных коразмерностей, начиная с 9-й) и в таблице 2 для четного $n$ (многоточие обозначает линейную комбиначию эйлеровых характеристик многообразий особенностей четных коразмерностей, начиная c 8-й). Аналогичные формуль для әллиптической компоненты $U$ см. в [5].

3) Если $n$ нечетное, то эйлерова характеристика $\chi(\Gamma)$ гиперповерхности Г является универсальной линейной комбинацией эйлеровых характеристик четномерных многообразий ее особенностей (коэффициенты этой комбинации не зависят от $n, V, \mathscr{F}$ и выбора компоненты $U)$. А именно, в случае 
гиперболической компоненты $U$

$$
\begin{aligned}
\chi(\Gamma)= & \chi\left(A_{1}\right)-\frac{1}{2}\left(\chi\left(3 A_{1}\right)+\chi\left(A_{2}+A_{1}\right)+\chi\left(A_{3}\right)\right)+\chi\left(5 A_{1}\right)+\chi\left(A_{2}+3 A_{1}\right) \\
& +\chi\left(2 A_{2}+A_{1}\right)+\chi\left(A_{3}+2 A_{1}\right)+\chi\left(A_{3}+A_{2}\right)+\chi\left(A_{4}+A_{1}\right)+\chi\left(A_{5}\right) \\
& -\frac{1}{4}\left(17 \chi\left(7 A_{1}\right)+17 \chi\left(A_{2}+5 A_{1}\right)+17 \chi\left(2 A_{2}+3 A_{1}\right)+17 \chi\left(3 A_{2}+A_{1}\right)\right. \\
& +17 \chi\left(A_{3}+4 A_{1}\right)+15 \chi\left(A_{3}+A_{2}+2 A_{1}\right)+15 \chi\left(A_{3}+2 A_{2}\right) \\
& +13 \chi\left(2 A_{3}+A_{1}\right)+17 \chi\left(A_{4}+3 A_{1}\right)+15 \chi\left(A_{4}+A_{2}+A_{1}\right)+16 \chi\left(A_{4}+A_{3}\right) \\
& \left.+17 \chi\left(A_{5}+2 A_{1}\right)+17 \chi\left(A_{5}+A_{2}\right)+15 \chi\left(A_{6}+A_{1}\right)+17 \chi\left(A_{7}\right)\right)+\cdots
\end{aligned}
$$

\begin{tabular}{|c|c|}
\hline \multirow[t]{2}{*}{$c=2$} & $\begin{array}{l}8 \chi\left(2 A_{1}\right)=12 \chi\left(3 A_{1}\right)+8 \chi\left(A_{2}+A_{1}\right)+4 \chi\left(A_{3}\right)-20 \chi\left(5 A_{1}\right)-18 \chi\left(A_{2}+3 A_{1}\right) \\
\quad-16 \chi\left(2 A_{2}+A_{1}\right)-16 \chi\left(A_{3}+2 A_{1}\right)-14 \chi\left(A_{3}+A_{2}\right)-14 \chi\left(A_{4}+A_{1}\right) \\
\quad-12 \chi\left(A_{5}\right)+84 \chi\left(7 A_{1}\right)+80 \chi\left(A_{2}+5 A_{1}\right)+76 \chi\left(2 A_{2}+3 A_{1}\right) \\
\quad+72 \chi\left(3 A_{2}+A_{1}\right)+76 \chi\left(A_{3}+4 A_{1}\right)+66 \chi\left(A_{3}+A_{2}+2 A_{1}\right) \\
\quad+62 \chi\left(A_{3}+2 A_{2}\right)+56 \chi\left(2 A_{3}+A_{1}\right)+72 \chi\left(A_{4}+3 A_{1}\right) \\
\quad+62 \chi\left(A_{4}+A_{2}+A_{1}\right)+61 \chi\left(A_{4}+A_{3}\right)+68 \chi\left(A_{5}+2 A_{1}\right) \\
\quad+64 \chi\left(A_{5}+A_{2}\right)+58 \chi\left(A_{6}+A_{1}\right)+60 \chi\left(A_{7}\right)+\cdots\end{array}$ \\
\hline & $\begin{array}{l}8 \chi\left(A_{2}\right)=4 \chi\left(A_{2}+A_{1}\right)+8 \chi\left(A_{3}\right)-2 \chi\left(A_{2}+3 A_{1}\right)-4 \chi\left(2 A_{2}+A_{1}\right) \\
\quad-4 \chi\left(A_{3}+2 A_{1}\right)-6 \chi\left(A_{3}+A_{2}\right)-6 \chi\left(A_{4}+A_{1}\right)-8 \chi\left(A_{5}\right) \\
\quad+4 \chi\left(A_{2}+5 A_{1}\right)+8 \chi\left(2 A_{2}+3 A_{1}\right)+12 \chi\left(3 A_{2}+A_{1}\right) \\
\quad+8 \chi\left(A_{3}+4 A_{1}\right)+10 \chi\left(A_{3}+A_{2}+2 A_{1}\right)+14 \chi\left(A_{3}+2 A_{2}\right) \\
\quad+12 \chi\left(2 A_{3}+A_{1}\right)+12 \chi\left(A_{4}+3 A_{1}\right)+14 \chi\left(A_{4}+A_{2}+A_{1}\right) \\
\quad+19 \chi\left(A_{4}+A_{3}\right)+16 \chi\left(A_{5}+2 A_{1}\right)+20 \chi\left(A_{5}+A_{2}\right) \\
\quad+18 \chi\left(A_{6}+A_{1}\right)+24 \chi\left(A_{7}\right)+\cdots\end{array}$ \\
\hline \multirow[t]{2}{*}{$c=4$} & $\begin{array}{l}4 \chi\left(4 A_{1}\right)=10 \chi\left(5 A_{1}\right)+4 \chi\left(A_{2}+3 A_{1}\right)+2 \chi\left(A_{3}+2 A_{1}\right)-35 \chi\left(7 A_{1}\right) \\
\quad-25 \chi\left(A_{2}+5 A_{1}\right)-16 \chi\left(2 A_{2}+3 A_{1}\right)-8 \chi\left(3 A_{2}+A_{1}\right)-19 \chi\left(A_{3}+4 A_{1}\right) \\
\quad-11 \chi\left(A_{3}+A_{2}+2 A_{1}\right)-4 \chi\left(A_{3}+2 A_{2}\right)-7 \chi\left(2 A_{3}+A_{1}\right) \\
\quad-13 \chi\left(A_{4}+3 A_{1}\right)-6 \chi\left(A_{4}+A_{2}+A_{1}\right)-3 \chi\left(A_{4}+A_{3}\right) \\
\quad-8 \chi\left(A_{5}+2 A_{1}\right)-2 \chi\left(A_{5}+A_{2}\right)-4 \chi\left(A_{6}+A_{1}\right)-\chi\left(A_{7}\right)+\cdots\end{array}$ \\
\hline & $\begin{array}{l}4 \chi\left(A_{2}+2 A_{1}\right)=6 \chi\left(A_{2}+3 A_{1}\right)+8 \chi\left(2 A_{2}+A_{1}\right)+4 \chi\left(A_{3}+2 A_{1}\right) \\
\quad+2 \chi\left(A_{3}+A_{2}\right)+4 \chi\left(A_{4}+A_{1}\right)-10 \chi\left(A_{2}+5 A_{1}\right) \\
\quad-18 \chi\left(2 A_{2}+3 A_{1}\right)-24 \chi\left(3 A_{2}+A_{1}\right)-12 \chi\left(A_{3}+4 A_{1}\right) \\
\quad-16 \chi\left(A_{3}+A_{2}+2 A_{1}\right)-20 \chi\left(A_{3}+2 A_{2}\right)-12 \chi\left(2 A_{3}+A_{1}\right) \\
\quad-15 \chi\left(A_{4}+3 A_{1}\right)-17 \chi\left(A_{4}+A_{2}+A_{1}\right)-14 \chi\left(A_{4}+A_{3}\right) \\
\quad-16 \chi\left(A_{5}+2 A_{1}\right)-18 \chi\left(A_{5}+A_{2}\right)-13 \chi\left(A_{6}+A_{1}\right)-12 \chi\left(A_{7}\right)+\cdots\end{array}$ \\
\hline
\end{tabular}

(многоточие обозначает то же самое, что в таблице 1). Аналогичная формула для әллиптической компоненты $U$ приведена в [5].

ТАБЛИцА 1: $n$ нечетное 
ТАБлицА 1 (продолжение)

\begin{tabular}{|c|c|}
\hline \multirow[t]{3}{*}{$c=4$} & $\begin{array}{l}4 \chi\left(2 A_{2}\right)=2 \chi\left(2 A_{2}+A_{1}\right)+4 \chi\left(A_{3}+A_{2}\right)+2 \chi\left(A_{5}\right)-\chi\left(2 A_{2}+3 A_{1}\right) \\
-3 \chi\left(3 A_{2}+A_{1}\right)-2 \chi\left(A_{3}+A_{2}+2 A_{1}\right)-5 \chi\left(A_{3}+2 A_{2}\right) \\
-4 \chi\left(2 A_{3}+A_{1}\right)-3 \chi\left(A_{4}+A_{2}+A_{1}\right)-6 \chi\left(A_{4}+A_{3}\right) \\
-\chi\left(A_{5}+2 A_{1}\right)-5 \chi\left(A_{5}+A_{2}\right)-3 \chi\left(A_{6}+A_{1}\right)-6 \chi\left(A_{7}\right)+\cdots\end{array}$ \\
\hline & $\begin{array}{l}2 \chi\left(A_{3}+A_{1}\right)=2 \chi\left(A_{3}+2 A_{1}\right)+2 \chi\left(A_{3}+A_{2}\right)+2 \chi\left(A_{4}+A_{1}\right)+2 \chi\left(A_{5}\right) \\
\quad-2 \chi\left(A_{3}+4 A_{1}\right)-2 \chi\left(A_{3}+A_{2}+2 A_{1}\right)-2 \chi\left(A_{3}+2 A_{2}\right) \\
\quad-4 \chi\left(2 A_{3}+A_{1}\right)-3 \chi\left(A_{4}+3 A_{1}\right)-3 \chi\left(A_{4}+A_{2}+A_{1}\right) \\
\quad-5 \chi\left(A_{4}+A_{3}\right)-4 \chi\left(A_{5}+A_{2}\right)-4 \chi\left(A_{5}+2 A_{1}\right) \\
\quad-5 \chi\left(A_{6}+A_{1}\right)-6 \chi\left(A_{7}\right)+\cdots\end{array}$ \\
\hline & $\begin{array}{l}4 \chi\left(A_{4}\right)=2 \chi\left(A_{4}+A_{1}\right)+4 \chi\left(A_{5}\right)-\chi\left(A_{4}+3 A_{1}\right)-\chi\left(A_{4}+A_{2}+A_{1}\right) \\
\quad-\chi\left(A_{4}+A_{3}\right)-2 \chi\left(A_{5}+2 A_{1}\right)-2 \chi\left(A_{5}+A_{2}\right) \\
\quad-3 \chi\left(A_{6}+A_{1}\right)-4 \chi\left(A_{7}\right)+\cdots\end{array}$ \\
\hline \multirow[t]{11}{*}{$c=6$} & $2 \chi\left(6 A_{1}\right)=7 \chi\left(7 A_{1}\right)+2 \chi\left(A_{2}+5 A_{1}\right)+\chi\left(A_{3}+4 A_{1}\right)+\cdots$ \\
\hline & $\begin{array}{l}2 \chi\left(A_{2}+4 A_{1}\right)=5 \chi\left(A_{2}+5 A_{1}\right)+4 \chi\left(2 A_{2}+3 A_{1}\right)+2 \chi\left(A_{3}+4 A_{1}\right) \\
\quad+\chi\left(A_{3}+A_{2}+2 A_{1}\right)+2 \chi\left(A_{4}+3 A_{1}\right)+\cdots\end{array}$ \\
\hline & $\begin{array}{l}2 \chi\left(2 A_{2}+2 A_{1}\right)=3 \chi\left(2 A_{2}+3 A_{1}\right)+6 \chi\left(3 A_{2}+A_{1}\right)+2 \chi\left(A_{3}+A_{2}+2 A_{1}\right) \\
\quad+\chi\left(A_{3}+2 A_{2}\right)+2 \chi\left(A_{4}+A_{2}+A_{1}\right)+\chi\left(A_{5}+2 A_{1}\right)+\cdots\end{array}$ \\
\hline & $2 \chi\left(3 A_{2}\right)=\chi\left(3 A_{2}+A_{1}\right)+2 \chi\left(A_{3}+2 A_{2}\right)+\chi\left(A_{5}+A_{2}\right)+\cdots$ \\
\hline & $\begin{array}{l}\chi\left(A_{3}+3 A_{1}\right)=2 \chi\left(A_{3}+4 A_{1}\right)+\chi\left(A_{3}+A_{2}+2 A_{1}\right)+\chi\left(2 A_{3}+A_{1}\right) \\
\quad+\chi\left(A_{4}+3 A_{1}\right)+\chi\left(A_{5}+2 A_{1}\right)+\cdots\end{array}$ \\
\hline & $\begin{array}{l}\chi\left(A_{3}+A_{2}+A_{1}\right)=\chi\left(A_{3}+A_{2}+2 A_{1}\right)+2 \chi\left(A_{3}+2 A_{2}\right) \\
\quad+2 \chi\left(2 A_{3}+A_{1}\right)+\chi\left(A_{4}+A_{2}+A_{1}\right)+\chi\left(A_{4}+A_{3}\right) \\
\quad+\chi\left(A_{5}+A_{2}\right)+\chi\left(A_{6}+A_{1}\right)+\cdots\end{array}$ \\
\hline & $2 \chi\left(2 A_{3}\right)=\chi\left(2 A_{3}+A_{1}\right)+2 \chi\left(A_{4}+A_{3}\right)+\chi\left(A_{7}\right)+\cdots$ \\
\hline & $\begin{array}{l}2 \chi\left(A_{4}+2 A_{1}\right)=3 \chi\left(A_{4}+3 A_{1}\right)+2 \chi\left(A_{4}+A_{2}+A_{1}\right) \\
\quad+\chi\left(A_{4}+A_{3}\right)+2 \chi\left(A_{5}+2 A_{1}\right)+2 \chi\left(A_{6}+A_{1}\right)+\cdots\end{array}$ \\
\hline & $\begin{array}{l}2 \chi\left(A_{4}+A_{2}\right)=\chi\left(A_{4}+A_{2}+A_{1}\right)+2 \chi\left(A_{4}+A_{3}\right) \\
\quad+2 \chi\left(A_{5}+A_{2}\right)+2 \chi\left(A_{7}\right)+\cdots\end{array}$ \\
\hline & $\chi\left(A_{5}+A_{1}\right)=\chi\left(A_{5}+2 A_{1}\right)+\chi\left(A_{5}+A_{2}\right)+\chi\left(A_{6}+A_{1}\right)+\chi\left(A_{7}\right)+\cdots$ \\
\hline & $2 \chi\left(A_{6}\right)=2 \chi\left(A_{7}\right)+\chi\left(A_{6}+A_{1}\right)+\cdots$ \\
\hline
\end{tabular}

Теорема 1.9 доказана в конце $\S 3$ (фактически указан простой комбинаторный алгоритм для вычисления всех соотношений (2) в любой размерности $n$ ). 
ТАБЛИЦА 2: $n$ четное

\begin{tabular}{|c|c|}
\hline$c=1$ & $\begin{array}{l}4 \chi\left(A_{1}\right)=4 \chi\left(2 A_{1}\right)+4 \chi\left(A_{2}\right)-4 \chi\left(4 A_{1}\right)-4 \chi\left(A_{2}+2 A_{1}\right)-4 \chi\left(2 A_{2}\right) \\
\quad-4 \chi\left(A_{3}+A_{1}\right)-4 \chi\left(A_{4}\right)+12 \chi\left(6 A_{1}\right)+12 \chi\left(A_{2}+4 A_{1}\right) \\
\quad+12 \chi\left(2 A_{2}+2 A_{1}\right)+12 \chi\left(3 A_{2}\right)+12 \chi\left(A_{3}+3 A_{1}\right) \\
\quad+11 \chi\left(A_{3}+A_{2}+A_{1}\right)+8 \chi\left(2 A_{3}\right)+12 \chi\left(A_{4}+2 A_{1}\right) \\
\quad+10 \chi\left(A_{4}+A_{2}\right)+12 \chi\left(A_{5}+A_{1}\right)+10 \chi\left(A_{6}\right)+\cdots\end{array}$ \\
\hline \multirow[t]{3}{*}{$c=3$} & $\begin{array}{l}4 \chi\left(A_{3}\right)=2 \chi\left(A_{3}+A_{1}\right)+4 \chi\left(A_{4}\right)-\chi\left(A_{3}+3 A_{1}\right) \\
-\chi\left(A_{3}+A_{2}+A_{1}\right)-2 \chi\left(2 A_{3}\right)-2 \chi\left(A_{4}+2 A_{1}\right) \\
-2 \chi\left(A_{4}+A_{2}\right)-3 \chi\left(A_{5}+A_{1}\right)-4 \chi\left(A_{6}\right)+\cdots\end{array}$ \\
\hline & $\begin{array}{l}4 \chi\left(A_{2}+A_{1}\right)=4 \chi\left(A_{2}+2 A_{1}\right)+8 \chi\left(2 A_{2}\right)+4 \chi\left(A_{3}+A_{1}\right)+4 \chi\left(A_{4}\right) \\
\quad-4 \chi\left(A_{2}+4 A_{1}\right)-8 \chi\left(2 A_{2}+2 A_{1}\right)-12 \chi\left(3 A_{2}\right)-6 \chi\left(A_{3}+3 A_{1}\right) \\
\quad-9 \chi\left(A_{3}+A_{2}+A_{1}\right)-8 \chi\left(2 A_{3}\right)-8 \chi\left(A_{4}+2 A_{1}\right) \\
\quad-10 \chi\left(A_{4}+A_{2}\right)-10 \chi\left(A_{5}+A_{1}\right)-10 \chi\left(A_{6}\right)+\cdots\end{array}$ \\
\hline & $\begin{array}{l}4 \chi\left(3 A_{1}\right)=8 \chi\left(4 A_{1}\right)+4 \chi\left(A_{2}+2 A_{1}\right)+2 \chi\left(A_{3}+A_{1}\right)-20 \chi\left(6 A_{1}\right) \\
\quad-16 \chi\left(A_{2}+4 A_{1}\right)-12 \chi\left(2 A_{2}+2 A_{1}\right)-8 \chi\left(3 A_{2}\right)-13 \chi\left(A_{3}+3 A_{1}\right) \\
\quad-9 \chi\left(A_{3}+A_{2}+A_{1}\right)-6 \chi\left(2 A_{3}\right)-10 \chi\left(A_{4}+2 A_{1}\right) \\
\quad-6 \chi\left(A_{4}+A_{2}\right)-7 \chi\left(A_{5}+A_{1}\right)-4 \chi\left(A_{6}\right)+\cdots\end{array}$ \\
\hline \multirow[t]{7}{*}{$c=5$} & $2 \chi\left(A_{5}\right)=\chi\left(A_{5}+A_{1}\right)+2 \chi\left(A_{6}\right)+\cdots$ \\
\hline & $2 \chi\left(A_{3}+A_{2}\right)=\chi\left(A_{3}+A_{2}+A_{1}\right)+4 \chi\left(2 A_{3}\right)+2 \chi\left(A_{4}+A_{2}\right)+2 \chi\left(A_{6}\right)+\cdots$ \\
\hline & $\chi\left(A_{4}+A_{1}\right)=\chi\left(A_{4}+2 A_{1}\right)+\chi\left(A_{4}+A_{2}\right)+\chi\left(A_{5}+A_{1}\right)+\chi\left(A_{6}\right)+\cdots$ \\
\hline & $\begin{array}{l}2 \chi\left(2 A_{2}+A_{1}\right)=2 \chi\left(2 A_{2}+2 A_{1}\right)+6 \chi\left(3 A_{2}\right)+2 \chi\left(A_{3}+A_{2}+A_{1}\right) \\
\quad+2 \chi\left(A_{4}+A_{2}\right)+\chi\left(A_{5}+A_{1}\right)+\cdots\end{array}$ \\
\hline & $\begin{array}{l}2 \chi\left(A_{3}+2 A_{1}\right)=3 \chi\left(A_{3}+3 A_{1}\right)+2 \chi\left(A_{3}+A_{2}+A_{1}\right)+2 \chi\left(2 A_{3}\right) \\
\quad+2 \chi\left(A_{4}+2 A_{1}\right)+2 \chi\left(A_{5}+A_{1}\right)+\cdots\end{array}$ \\
\hline & $\begin{array}{l}2 \chi\left(A_{2}+3 A_{1}\right)=4 \chi\left(A_{2}+4 A_{1}\right)+4 \chi\left(2 A_{2}+2 A_{1}\right)+2 \chi\left(A_{3}+3 A_{1}\right) \\
\quad+\chi\left(A_{3}+A_{2}+A_{1}\right)+2 \chi\left(A_{4}+2 A_{1}\right)+\cdots\end{array}$ \\
\hline & $2 \chi\left(5 A_{1}\right)=6 \chi\left(6 A_{1}\right)+2 \chi\left(A_{2}+4 A_{1}\right)+\chi\left(A_{3}+3 A_{1}\right)+\cdots$ \\
\hline
\end{tabular}

В настоящей работе также доказано, что в условиях теоремы 1.9 не сушествует универсальных линейных соотношений (с вешественными коэффициентами) между эйлеровьми характеристиками многообразий особенностей гиперповерхности Г, которые не были бы линейньми комбинациями соотношений вида (2).

А именно, составим список $\mathscr{A}^{1}, \ldots, \mathscr{A}^{N}$ всех (попарно не диффеоморфных) устойчивых особенностей коранга 1 , которые могут быть у фронтов в гладком $n$-мерном многообразии и коразмерность которых сравнима с $n$ по модулю 2 (многообразия таких особенностей имеют четную размерность). Через $N_{\text {odd }}$ обозначим количество особенностей в списке $\mathscr{A}^{1}, \ldots, \mathscr{A}^{N}$, которые имеют только нечетные кратности. 
Рассмотрим произвольный неприводимый компактный устойчивый фронт $\mathscr{F}$ коранга 1 в $\mathbb{R}^{n}$ и произвольную ограниченную правильную связную компоненту дополнения $\mathbb{R}^{n} \backslash \mathscr{F}$. Краю Г этой компоненты сопоставим вектор

$$
v(\Gamma)=\left(\chi\left(\mathscr{A}_{\Gamma}^{1}\right), \ldots, \chi\left(\mathscr{A}_{\Gamma}^{N}\right)\right) \in \mathbb{R}^{N}
$$

из эйлеровых характеристик $\chi\left(\mathscr{A}_{\Gamma}^{i}\right)$ многообразий $\mathscr{A}_{\Gamma}^{i}$ особенностей $\mathscr{A}^{i}, i=$ $1, \ldots, N$, гиперповерхности $\Gamma$.

Пусть $G_{\text {hyp }}\left(G_{\text {elp }}\right)$ - подмножество в $\mathbb{R}^{N}$, образованное векторами $v(\Gamma)$ для всех фронтов $\mathscr{F} \subset \mathbb{R}^{n}$ указанного типа и для всех гиперболических (эллиптических соответственно) ограниченных связных компонент их дополнений $\mathbb{R}^{n} \backslash \mathscr{F}$.

Теорема 1.10. Eсли $n>1$, то множсество $G_{\text {hyp }}\left(G_{\text {elp }}\right)$ содержит полугруппу с $N$ ( $N_{\text {odd }}$ соответственно) линейно независимыми образующими.

При $n=1$ граница любой связной компоненты дополнения к фронту состоит из одной или двух точек - особенностей $A_{1}$.

Следует отметить, что эйлеровы характеристики многообразий особенностей границы связной компоненты дополнения к фронту ограничены также условиями типа неравенств, четности и т.п. Однако мы не знаем всех этих условий.

Доказательство теоремы 1.10 приведено в $\S 9$.

\section{§2. Характеристические многообразия и отображения особенностей фронта}

Доказательство теоремы 1.9 основано на разрешении особенностей устойчивого фронта коранга 1. Это разрешение было описано в работе [4]. Здесь приведены основные элементы этой конструкции.

Пусть $V$ - гладкое $n$-мерное многообразие. Рассмотрим пространство $P T^{*} V$ проективизации кокасательного расслоения многообразия $V$. Это пространство образовано контактными әлементами $(\pi, x)$, где $x \in V$, а $\pi \subset T_{x} V$ - касательная гиперплоскость в точке $x$.

Пространство $P T^{*} V$ является гладким $(2 n-1)$-мерным многообразием и обладает естественной контактной структурой (см. [1]). Проекция $\rho: P T^{*} V \rightarrow V$ является лежандровым расслоением относительно этой структуры.

Пусть $\mathscr{F}$ - устойчивый фронт коранга 1 в многообразии $V$ (можно взять даже произвольную гиперповерхность с особенностями типа $\left.A_{\mu_{1}}+\cdots+A_{\mu_{m}}\right)$. Рассмотрим замыкание $L$ подмножества в $P T^{*} V$, образованного контактными элементами на $V$, которые касаются фронта $\mathscr{F}$ в его гладких точках.

Множество $L$ является гладким $(n-1)$-мерньм лежандровым подмногообразием в пространстве $P T^{*} V$. Сужение проекции $\rho$ на $L$ является устойчивым лежандровым отображением $f: L \rightarrow V$ коранга 1. Гиперповерхность $\mathscr{F}$ является фронтом этого отображения.

Теорема 2.1 [4]. Имеется конструкиия, которая фронту भ्F и всякой особенности $\mathscr{A}=A_{\mu_{1}}+\cdots+A_{\mu_{m}}$ такой, что многоообразие $\mathscr{A}_{\mathscr{F}}$ особенностей $\mathscr{A}$ 
фронта $\mathscr{F}$ непусто, сопоставляет гладкое многообразие $\Phi_{\mathscr{A}}$ и гладкое собственное отображсение $\varphi_{\mathscr{A}}: \Phi_{\mathscr{A}} \rightarrow V$, обладающие следующими свойствами:

1) образом отображсения $\varphi_{\mathscr{A}}$ является замыкание $\overline{\mathscr{A}}_{\mathscr{F}} \subset V$ многообразия $\mathscr{A}_{\mathscr{F}} ;$

2) если особенность $X=A_{\nu_{1}}+\cdots+A_{\nu_{p}}$ примыкает к особенности $\mathscr{A}$, m.е. $X_{\mathscr{F}} \subseteq \overline{\mathscr{A}}_{\mathscr{F}}$, то отображение $\varphi_{\mathscr{A}}$ является конечным накрытием над многообразием $X_{\mathscr{F}} ;$ кратность этого накрытия зависит только от классов особенностей $\mathscr{A}, X$ и не зависит от $n, V$ и $\mathscr{F}$.

Конструкция, о которой идет речь, описана ниже. Она называется разрешением особенностей устойчивого фронта коранга 1. Конструкция универсальна в том смысле, что она не зависит ни от фронта, ни от объемлющего его пространства (в том числе от размерности этого пространства).

ОПРЕДЕЛЕНИЕ 2.2. Многообразие $\Phi_{\mathscr{A}}$ и отображение $\varphi_{\mathscr{A}}$, получающиеся в результате разрешения особенности $\mathscr{A}$ фронта $\mathscr{F}$, называются характеристическими для этой особенности. Кратность отображения $\varphi_{\mathscr{A}}$ над многообразием $X_{\mathscr{F}}$ особенностей $X$ фронта $\mathscr{F}$ назьвается индексом особенности $X$ относительно особенности $\mathscr{A}$ и обозначается через $I_{\mathscr{A}}(X)$.

Характеристические многообразия и отображения особенностей фронта $\mathscr{F}$ строятся индуктивно. А именно, упорядочим произвольным образом набор кратностей $\mu_{1}, \ldots, \mu_{m}$ особенности $\mathscr{A}$. Тогда отображение $\varphi_{\mathscr{A}}$ является композицией гладких отображений

$$
\Phi_{A_{\mu_{1}}}+\cdots+A_{\mu_{m}} \stackrel{\psi_{m}}{\longrightarrow} \cdots \stackrel{\psi_{3}}{\longrightarrow} \Phi_{A_{\mu_{1}}+A_{\mu_{2}}} \stackrel{\psi_{2}}{\longrightarrow} \Phi_{A_{\mu_{1}}} \stackrel{\psi_{1}}{\longrightarrow} V .
$$

Каждое отображение $\psi_{i}$ является, в свою очередь, композицией гладких отображений

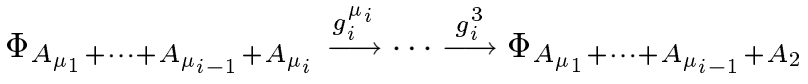

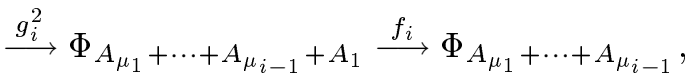

где $f_{i}$ - устойчивое лежандрово отображение коранга 1 , а $g_{i}^{j}$ - вложение коразмерности 1 гладкого замыкания множества особенностей $A_{j}$ отображения $f_{i}$.

В качестве $f_{1}: \Phi_{A_{1}} \rightarrow \Phi_{\emptyset}$ выступает исходное лежандрово отображение $f$, так что $\Phi_{A_{1}}=L, \Phi_{\emptyset}=V, \varphi_{A_{1}}=f$. Многообразие $\Phi_{A_{\mu_{1}}} \subseteq \Phi_{A_{1}}$ - гладкое замькание множества особенностей $A_{\mu_{1}}$ отображения $f_{1}$. Лежандрово отображение $f_{2}: \Phi_{A_{\mu_{1}}}+A_{1} \rightarrow \Phi_{A_{\mu_{1}}}$ определяется следуюшим образом.

Пусть $\mathscr{F}^{1}$ - замыкание в многообразии $\Phi_{A_{\mu_{1}}}$ множества точек $\xi$ таких, что фронт $\mathscr{F}^{0}=\mathscr{F}$ имеет в точке $f_{1}(\xi)$ особенность $A_{\mu_{1}}+A_{1}$. Если множество $\mathscr{F}^{1}$ непусто, то оно является устойчивьм фронтом коранга 1 в $\Phi_{A_{\mu_{1}}}$ (см. [4]). Фронт $\mathscr{F} 1$ называется $A_{\mu_{1}}$-преобразованием фронта $\mathscr{F}^{0}$ и обозначается через $\left[A_{\mu_{1}}\right]\left(\mathscr{F}^{0}\right)$.

По определению многообразие $\Phi_{A_{1}}+A_{1} \subset P T^{*}\left(\Phi_{A_{\mu_{1}}}\right)$ является замыканием множества контактных элементов, касающихся фронта $\mathscr{F}^{1}$ в его гладких точках. Лежандровым отображением $f_{2}$ является сужение естественной проекции $P T^{*}\left(\Phi_{A_{\mu_{1}}}\right) \rightarrow \Phi_{A_{\mu_{1}}}$ на $\Phi_{A_{\mu_{1}}+A_{1}}$. 
Многообразие $\Phi_{A_{\mu_{1}}}+\cdots+A_{\mu_{i}}+A_{1}$ и лежандрово отображение $f_{i+1}, i>1$, строятся аналогично при помощи фронта

$$
\mathscr{F}^{i}=\left[A_{\mu_{i}}\right]\left(\mathscr{F}^{i-1}\right) \subset \Phi_{A_{\mu_{1}}+\cdots+A_{\mu_{i}}}
$$

$\Phi$ ронт $\mathscr{F}^{m}$ называется $\mathscr{A}$-преобразованием фронта $\mathscr{F}$ и обозначается $[\mathscr{A}](\mathscr{F})$.

ЗАмечАниЕ 2.3. Разрешение особенностей фронтов, описанное выше, обобщает принцип итерации Клеймана [7]. Этот принцип обычно используется в комплексных задачах при исследовании циклов кратных точек общих голоморфных отображений коранга 1 (см., например, [8] и [9]). Мы же изучаем циклы произвольных устойчивых мультиособенностей гладких (лежандровых) отображений коранга 1.

ПРЕДЛОЖЕНИЕ 2.4. Для любого $\mathscr{A}=A_{\mu_{1}}+\cdots+A_{\mu_{m}}$ многообразие $\Phi_{\mathscr{A}}$ диффеоморфно гладкому замыканию в $(L)^{m}=L \times \cdots \times L$ (m раз) многообразия $L_{\mathscr{A}}$ наборов $\left(\left(\pi_{1}, x_{1}\right), \ldots,\left(\pi_{m}, x_{m}\right)\right)$ контактных әлементов на $V$ таких, что $x_{1}=\cdots=x_{m}=x$, гиперплоскости $\pi_{1}, \ldots, \pi_{m}$ пересекаются в точке $x$ трансверсально, а отображсение $f$ имеет особенность $A_{\mu_{i}}$ в точке $\left(\pi_{i}, x_{i}\right)$, $i=1, \ldots, m$.

ДоКАЗАТЕЛЬСТво. При $m=1$ это хорошо известный факт (он уже использовался выше при построении вложений $g_{i}^{j}$ ). Предположим, что это утверждение верно при всех $m \leqslant m_{0}-1$, и докажем его для $m=m_{0}$. Для упрошения обозначений мы опустим индекс 0 у числа $m_{0}$ и положим $A_{\mu_{1}}+\cdots+A_{\mu_{m-1}}=\widetilde{\mathscr{A}}$.

Пусть П - гладкое многообразие, образованное наборами $\left(\pi_{1}, \ldots, \pi_{m-1}, \tau, x\right)$ такими, что $x$ - точка многообразия $V, \pi_{1}, \ldots, \pi_{m-1}$-гиперплоскости в касательном пространстве к $V$ в точке $x$, а $\tau$ - подпространство коразмерности $\mu_{1}+\cdots+$ $\mu_{m-1}+1$ в $T_{x} V$. Тогда многообразие $P T^{*}\left(L_{\tilde{A}}\right)$ диффеоморфно подмногообразию в П, образованному наборами $\left(\pi_{1}, \ldots, \pi_{m-1}, \tau, x\right)$ такими, что $x \in \tilde{\mathscr{A}}_{\mathscr{F}}$, лежандрово отображение $f: L \rightarrow V$ имеет особенность $A_{\mu_{i}}$ в точке $\left(\pi_{i}, x\right), i=1, \ldots, m-1$,

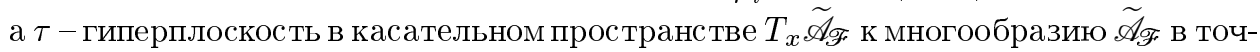
ке $x$.

Следовательно, многообразие $\varphi_{\mathscr{A}}^{-1}\left(\mathscr{A}_{\mathscr{F}}\right) \subseteq \Phi_{\mathscr{A}}$ дифффеоморфно подмногообразию в П, образованному наборами $\left(\pi_{1}, \ldots, \pi_{m-1}, \tau, x\right)$ такими, что $x \in \mathscr{A}_{\mathscr{F}}, \tau=$ $\pi_{m} \cap T_{x} \widetilde{\mathscr{A}}_{\widetilde{F}}$, лежандрово отображение $f: L \rightarrow V$ имеет особенность $A_{\mu_{j}}$ в точке $\left(\pi_{j}, x\right), j=1, \ldots, m$, a $\widetilde{F}=\bigcup_{i=1}^{m-1} f\left(L,\left(\pi_{i}, x\right)\right)$, где $f\left(L,\left(\pi_{i}, x\right)\right)$ - фронт ростка многообразия $L$ в точке $\left(\pi_{i}, x\right)$. Это подмногообразие, в свою очередь, диффеоморфно подмногообразию $L_{\mathscr{A}} \subset(L)^{m}$.

Остается лиш проверить, что построенньй нами диффеоморфизм многообразий $\varphi_{\mathscr{A}}^{-1}\left(\mathscr{A}_{\mathscr{F}}\right)$ и $L_{\mathscr{A}}$ продолжается до диффеоморфизма многообразия $\Phi_{\mathscr{A}}$ на гладкое замыкание подмногообразия $L_{\mathscr{A}}$ в многообразии $(L)^{m}$. Это сделано ниже в предложении 6.2 (утверждение 1)) путем непосредственных вычислений в локальных координатах. Предложение 2.4 доказано.

ПРЕДЛОЖЕНИЕ 2.5. Характеристические многообразия $\Phi_{\mathscr{A}}$, полученнье при разных упорядочениях набора кратностей особенности $\mathscr{A}$, естественно диффеоморфны. А именно, если $\widetilde{\Phi}_{\mathscr{A}}$ и $\widetilde{\varphi}_{\mathscr{A}}$ - характеристическое многообразие и характеристическое отображение особенности $\mathscr{A}$, соответствующие 
другому упорядочению набора кратностей этой особенности, то существует диффеоморфизм $\phi: \Phi_{\mathscr{A}} \rightarrow \widetilde{\Phi}_{\mathscr{A}}$ такой, что диаграмма

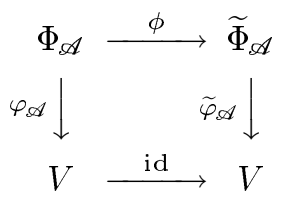

коммутативна.

Это утверждение следует из предложения 2.4. Действие диффеоморфизма $\phi$ состоит в перестановке сомножителей в произведении $(L)^{m}$.

ТеОрема 2.6 [4]. Индекс $I_{\mathscr{A}}(X)$ особенности $X=A_{\nu_{1}}+\cdots+A_{\nu_{p}}$ фронта $\mathscr{F}$ относительно особенности $\mathscr{A}=A_{\mu_{1}}+\cdots+A_{\mu_{m}}$ вычисляется рекуррентно исходя из следующих условий:

1) если $\mu^{*}=\max \left\{\mu_{1}, \ldots, \mu_{m}\right\}>\nu^{*}=\max \left\{\nu_{1}, \ldots, \nu_{p}\right\}$, mo $I_{\mathscr{A}}(X)=0$;

2) если $\mu^{*} \leqslant \nu^{*}$, mo

$I_{\mathscr{A}}(X)=\sum_{\nu_{i}=\mu^{*}, \mu^{*}+1} I_{\mathscr{A}-A_{\mu^{*}}}\left(X-A_{\nu_{i}}\right)+\sum_{\nu_{i}>\mu^{*}+1} I_{\mathscr{A}-A_{\mu^{*}}}\left(X-A_{\nu_{i}}+A_{\nu_{i}-\mu^{*}-1}\right)$, где $I_{\emptyset}(Y)=1$ для любого $Y$.

Для особенностей $\mathscr{A}=A_{\mu_{1}}+\cdots+A_{\mu_{m}}$ и $X=A_{\nu_{1}}+\cdots+A_{\nu_{p}}$ малых коразмерностей индекс $I_{\mathscr{A}}(X)$ можно быстро вычислить при помощи дерева (упорядоченной) пары особенностей $\mathscr{A} \vdash X$ в предположении, что $\mu_{1} \geqslant \cdots \geqslant \mu_{m}$. Это ориентированное дерево, ребра которого снабжены целыми положительными весами. Вершины обозначаются классами особенностей устойчивых фронтов коранга 1 или символом $\emptyset$. Корнем является особенность $X$.

Ориентации ребер определяются ориентациями цепей, выходящих из $X$ и заканчивающихся в концевых вершинах дерева. Длина любой такой цепи (т.е. количество ее ребер) не превосходит числа $m$ неприводимых компонент особенности $\mathscr{A}$. Вершины, находящиеся на расстоянии $i \leqslant m$ от корня $X$, а также ребра, в них входящие (вместе с весами), определяются вершинами, удаленными от $X$ на расстояние $i-1$.

А именно, из вершины $\emptyset$ ребра не выходят. Чтобы перечислить ребра, выходяшие из произвольной вершины $Y=A_{\varkappa_{1}}+\cdots+A_{\varkappa_{l}}$, обозначим через $a_{\varkappa}$ количество индексов $j \in\{1, \ldots, l\}$ таких, что $\varkappa_{j}=\varkappa$.

Пусть вершина $Y$ находится на расстоянии $i-1$ от корня $X$. Тогда:

1) если $a_{\mu_{i}}>0$, то из вершины $Y$ выходит ребро с концом $Y-A_{\varkappa_{i}}$, вес этого ребра равен $a_{\mu_{i}}$

2 ) если $a_{\mu_{i}+1}>0$, то из вершины $Y$ выходит ребро с концом $Y-A_{\mu_{i}+1}$, его вес равен $a_{\mu_{i}+1}$;

3 ) для любого натурального $\varkappa>\mu_{i}+1$ такого, что $a_{\varkappa}>0$, из вершины $Y$ выходит ребро с концом $Y-A_{\varkappa}+A_{\varkappa-\mu_{i}-1}$, его вес равен $a_{\varkappa}$.

Никакие другие ребра из вершины $Y$ не выходят. 
ОПРЕДЕЛЕНИЕ 2.7. Индексом чепи дерева пары особенностей $\mathscr{A} \vdash X$ называется произведение весов ребер, составляюших эту цепь.

Из теоремы 2.6 очевидно следует

Tеорема 2.8. Индекс $I_{\mathscr{A}}(X)$ особенности $X$ относительно особенности $\mathscr{A}$ равен сумме индексов всех чепей дерева пары $\mathscr{A} \vdash X$, которые выходят из корня $X$ и имеют длину, равную числу $m$ неприводимых компонент особенности $\mathscr{A}$.

ПримеР 2.9. На рис. 1 представлено дерево пары особенностей $\left(A_{2}+3 A_{1}\right) \vdash$ $\left(A_{3}+A_{2}+A_{1}\right)$. Вершины изображены прямоугольниками, ребра - стрелками, рядом с которыми написаны их веса. Дерево имеет две цепи длины 4, выходящие из корня $A_{3}+A_{2}+A_{1}$. Их индексы равны $1 \cdot 1 \cdot 1 \cdot 1=1$ и $1 \cdot 1 \cdot 2 \cdot 1=2$ соответственно. Поэтому

$$
I_{A_{2}+3 A_{1}}\left(A_{3}+A_{2}+A_{1}\right)=1+2=3 .
$$

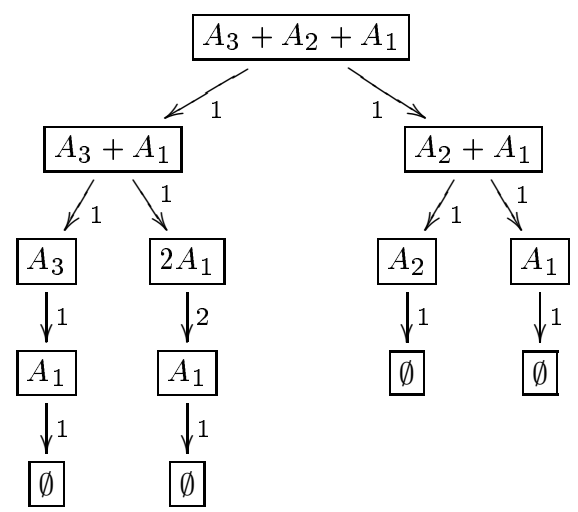

Рис. 1. Дерево пары особенностей $\left(A_{2}+3 A_{1}\right) \vdash\left(A_{3}+A_{2}+A_{1}\right)$

ПримеР 2.10. На рис. 2 представлено дерево пары особенностей $\left(A_{3}+3 A_{1}\right) \vdash$ $\left(2 A_{5}+A_{1}\right)$. Оно имеет шесть цепей длины 4 , выходящих из корня $2 A_{5}+A_{1}$. Несложньй подсчет показьвает, что

$I_{A_{3}+3 A_{1}}\left(2 A_{5}+A_{1}\right)=2 \cdot 2 \cdot 1 \cdot 1+2 \cdot 2 \cdot 1 \cdot 1+2 \cdot 2 \cdot 1 \cdot 1+2 \cdot 1 \cdot 2 \cdot 1+2 \cdot 1 \cdot 2 \cdot 1+2 \cdot 1 \cdot 1 \cdot 3=26$.

ПримеР 2.11. Дерево пары особенностей $m A_{1} \vdash k A_{1}$, где $m \leqslant k$, является цепю длины $m$, выходящей из корня $k A_{1}$. Веса ребер этой цепи изменяются на 1 при переходе через каждую вершину и пробегают все значения от $k$ до $k-m+1$. Поэтому

$$
I_{m A_{1}}\left(k A_{1}\right)=m !\left(\begin{array}{c}
k \\
m
\end{array}\right) .
$$

Используя указанный метод, нетрудно убедиться, например, в справедливости следуюших формул:

$$
I_{m A_{1}}\left(A_{2}+k A_{1}\right)=m !\left(\begin{array}{c}
k+1 \\
m
\end{array}\right)
$$




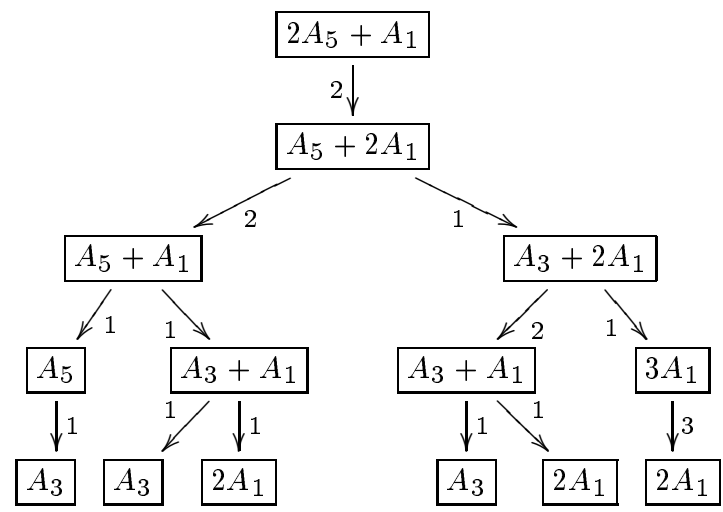

Рис. 2. Дерево пары особенностей $\left(A_{3}+3 A_{1}\right) \vdash\left(2 A_{5}+A_{1}\right)$

(этот индекс не равен 0 тогда и только тогда, когда $m \leqslant k+1$ );

$$
I_{m A_{1}}\left(A_{3}+k A_{1}\right)=\frac{m !}{2}\left(\left(\begin{array}{c}
k+2 \\
m
\end{array}\right)+\left(\begin{array}{l}
k \\
m
\end{array}\right)\right)
$$

(этот индекс не равен 0 тогда и только тогда, когда $m \leqslant k+2$ ).

\section{§3. Каноническая стратификация характеристического многообразия}

Пусть $\mathscr{A}=A_{\mu_{1}}+\cdots+A_{\mu_{m}}-$ произвольная особенность устойчивого фронта $\mathscr{F}$ коранга 1 в гладком $n$-мерном многообразии $V$. Рассмотрим характеристическое многообразие $\Phi_{\mathscr{A}}$ и характеристическое отображение $\varphi_{\mathscr{A}}: \Phi_{\mathscr{A}} \rightarrow V$ этой особенности.

ОПРЕДЕЛЕНИЕ 3.1. Канонической стратификацией многообразия $\Phi_{\mathscr{A}}$ называется разбиение этого многообразия на связные компоненты (страты) полных прообразов $\varphi_{\mathscr{A}}^{-1}\left(X_{\mathscr{F}}\right)$ многообразий $X_{\mathscr{F}}$ особенностей $X$ фронта $\mathscr{F}$, примыкающих к $\mathscr{A}$.

Каноническая стратификация многообразия $\Phi_{\mathscr{A}}$ является, очевидно, его $C^{\infty}$ стратификацией. Остов коразмерности $k$ этой стратификации (т.е. объединение стратов коразмерности не меньше $k$ ) мы будем обозначать через $\operatorname{St}_{k}\left(\Phi_{\mathscr{A}}\right)$.

ТЕОРемА 3.2. Каноническая стратификация многообразия $\Phi_{\mathscr{A}}$ является его $C^{\infty}$-стратификацией Уитни.

Чтобы доказать это утверждение, необходимо классифицировать особенности гиперповерхности $\operatorname{St}_{1}\left(\Phi_{\mathscr{A}}\right)$ относительно диффеоморфизмов объемлющего многообразия $\Phi_{\mathscr{A}}$. Такая классификация дается теоремой 4.7 , сформулированной в $\S 4$. Теорема 3.2 следует из теоремы 4.7 на основании предложения 4.6.

Пусть $\Gamma$ - край правильной связной компоненты дополнения $V \backslash \mathscr{F}$. Рассмотрим многообразие $\mathscr{A}_{\Gamma}$ особенностей $\mathscr{A}$ гиперповерхности Г и его замькание $\overline{\mathscr{A}}_{\Gamma} \subset V$. Обозначим через $\Phi_{\mathscr{A}}^{\Gamma}$ полньй прообраз множества $\overline{\mathscr{A}}_{\Gamma}$ при отображении $\varphi_{\mathscr{A}}$. 
Теорема 3.3. Если множсество $\overline{\mathscr{A}}_{\Gamma}$ компактно, то әйлерова характеристика $\chi\left(\Phi_{\mathscr{A}}^{\Gamma}\right)$ множества $\Phi_{\mathscr{A}}^{\Gamma}$ вычисляется по формуле

$$
(-1)^{n} \chi\left(\Phi_{\mathscr{A}}^{\Gamma}\right)=\sum_{X: X_{\Gamma} \subseteq \overline{\mathscr{A}}_{\Gamma}}(-1)^{\operatorname{codim} X} I_{\mathscr{A}}(X) \chi\left(X_{\Gamma}\right) .
$$

Это утверждение следует из теорем 2.1, 3.2 и правила вычисления эйлеровой характеристики компактного множества, являющегося объединением стратов $C^{\infty}$ стратификации Уитни гладкого замкнутого многообразия (см., например, [10]).

ОПРЕДЕЛЕНИЕ 3.4. Множество

$$
\partial_{\text {out }}\left(\mathscr{A}_{\mathscr{F}}\right)={\overline{\left(\mathscr{A}+A_{1}\right)_{\mathscr{F}}}} \subset V
$$

называется внешней гранищей многообразия $\mathscr{A}_{\mathscr{F}}$ особенностей $\mathscr{A}$ фронта $\mathscr{F}$. Множество

$$
C_{\text {in }}\left(\mathscr{A}_{\mathscr{F}}\right)=\overline{\mathscr{A}}_{\mathscr{F}} \backslash \partial_{\text {out }}\left(\mathscr{A}_{\mathscr{F}}\right)
$$

называется внутренним замыканием многообразия $\mathscr{A}_{\mathscr{F}}$.

Следующее утверждение доказано в $\S 7$.

ТЕОремА 3.5. Множсетво $\Phi_{\mathscr{A}}^{\Gamma}$ является многообразием с краем. Каждая связная компонента әтого многообразия представляет собой замыкание правильной связной компоненты дополнения $\Phi_{\mathscr{A}} \backslash \varphi_{\mathscr{A}}^{-1}\left(\partial_{\mathrm{out}}\left(\mathscr{A}_{\mathscr{F}}\right)\right)$.

Рассмотрим множества

$$
\partial_{\text {out }}\left(\mathscr{A}_{\Gamma}\right)=\partial_{\text {out }}\left(\mathscr{A}_{\mathscr{F}}\right) \cap \Gamma, \quad C_{\text {in }}\left(\mathscr{A}_{\Gamma}\right)=C_{\text {in }}\left(\mathscr{A}_{\mathscr{F}}\right) \cap \Gamma
$$

Они называются внешней границей и внутренним замыканием многообразия $\mathscr{A}_{\Gamma}$ особенностей $\mathscr{A}$ әиперповерхности Г (соответственно).

ТЕОРема 3.6. Пусть Г - край правильной связной компоненты дополнения $\kappa$ фронту Ғ общего положения в гладком п-мерном многообразии V. Предпо-

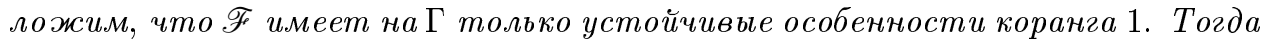
для любого $\mathscr{A}=A_{\mu_{1}}+\cdots+A_{\mu_{m}}$ такого, что многообразие $\mathscr{A}_{\Gamma}$ особенностей $\mathscr{A}$ гиперповерхности Г имеет нечетную размерность, а его замыкание $\overline{\mathscr{A}}_{\Gamma} \subset V$ компактно, справедливо равенство

$$
\begin{aligned}
\sum_{X: X_{\Gamma} \subseteq C_{\text {in }}\left(\mathscr{A}_{\Gamma}\right)}(-1)^{\operatorname{codim} X} I_{\mathscr{A}}(X) \chi\left(X_{\Gamma}\right) & \\
& =-\frac{1}{2} \sum_{X: X_{\Gamma} \subseteq \partial_{\text {out }}\left(\mathscr{A}_{\Gamma}\right)}(-1)^{\operatorname{codim} X} I_{\mathscr{A}}(X) \chi\left(X_{\Gamma}\right) .
\end{aligned}
$$

Действительно, без ограничения общности можно считать, что Г - край правильной связной компоненты дополнения к устойчивому фронту коранга 1 в $V$. Поэтому теорема 3.6 следует из теорем $3.3,3.5$ и того факта, что эйлерова характеристика нечетномерного компактного многообразия с краем равна половине эйлеровой характеристики края. 
ЗАмЕчАниЕ 3.7. В случае, когда гиперповерхность Г является краем эллиптической связной компоненты дополнения $V \backslash \mathscr{F}$, теорема 3.6 была сформулирована в работе [5]. В этом случае множество $C_{\text {in }}\left(\mathscr{A}_{\Gamma}\right)$ состоит из многообразий $X_{\Gamma}$ особенностей $X$ гиперповерхности $\Gamma$, у которых $\operatorname{deg} X=\operatorname{deg} \mathscr{A} ;$ множество $\partial_{\text {out }}\left(\mathscr{A}_{\Gamma}\right)$ состоит из многообразий $X_{\Gamma}$ особенностей $X$ таких, что $\operatorname{deg} X>\operatorname{deg} \mathscr{A}$.

Следуюшее утверждение хорошо известно.

ПРЕДЛОЖЕНИЕ 3.8. Пусть Г - край әиперболической или әллиптической связной компоненты $U$ дополнения $V \backslash \mathscr{F}$ к устойчивому фронту $\mathscr{F}$ коранга 1 в многообразии $V$. Тогда замыкание $\overline{\mathscr{A}}_{\Gamma}$ многообразия $\mathscr{A}_{\Gamma}$ особенностей $\mathscr{A}=$ $A_{\mu_{1}}+\cdots+A_{\mu_{m}}$ әтой гиперповерхности вычисляется рекуррентно исходя из следующих формул:

$$
\begin{aligned}
\overline{\mathscr{A}}_{\Gamma}=\mathscr{A}_{\Gamma} \cup \overline{\left(\mathscr{A}+A_{1}\right)_{\Gamma}} & \cup\left(\bigcup_{i=1}^{m} \overline{\left(\mathscr{A}-A_{\mu_{i}}+A_{\mu_{i}+1}\right)_{\Gamma}}\right) \\
& \cup\left(\bigcup_{1 \leqslant i<j \leqslant m} \overline{\left(\mathscr{A}-A_{\mu_{i}}-A_{\mu_{j}}+A_{\mu_{i}+\mu_{j}+1}\right)_{\Gamma}}\right),
\end{aligned}
$$

если компонента $U$ гиперболическая, $и$

$$
\left.\overline{\mathscr{A}}_{\Gamma}=\mathscr{A}_{\Gamma} \cup \overline{\left(\mathscr{A}+A_{1}\right)_{\Gamma}} \cup\left(\bigcup_{1 \leqslant i<j \leqslant m} \overline{\left(\mathscr{A}-A_{\mu_{i}}-A_{\mu_{j}}+A_{\mu_{i}+\mu_{j}+1}\right)}\right)_{\Gamma}\right),
$$

если компонента $U$ әллиптическая.

ДокАЗАТЕЛЬство. Зафиксируем целое неотрицательное $k$ и рассмотрим пространство многочленов вида $P(t)=Q(t) \prod_{i=1}^{m}\left(t-t_{i}\right)^{\mu_{i}+1}$, где $t_{1}, \ldots, t_{m}-$ произвольные вещественные числа, а $Q(t)=t^{k}+q_{k-1} t^{k-1}+\cdots+q_{0}-$ произвольный многочлен с вешественными коэффициентами такой, что $q_{k-1}=\sum_{i=1}^{m}\left(\mu_{i}+1\right) t_{i}$ (т.е. сумма всех корней многочлена $P$ равна нулю).

В общей точке любой кривой $\gamma$ общего положения в этом пространстве числа $t_{1}, \ldots, t_{m}$ попарно различны, а все корни многочлена $Q$ простые и не совпадают с $t_{1}, \ldots, t_{m}$. Но в некоторых (изолированных) точках кривой $\gamma$ кратности вешественных корней многочлена $P$ могут измениться, причем только одним из трех способов. А именно, либо многочлен $Q$ имеет двукратньй вешественный корень (отличный от $\left.t_{1}, \ldots, t_{m}\right)$, либо простой корень многочлена $Q$ совпадает с одним из чисел $t_{1}, \ldots, t_{m}$, либо $t_{i}=t_{j}$ для некоторых $i \neq j$.

Отсюда следует первая из указанных выше формул. Вторая формула получается аналогично, если рассмотреть пространство неотрицательных многочленов вида $P(t)$. Предложение 3.8 доказано.

ПримеР 3.9. Рассмотрим край Г эллиптической связной компоненты дополнения к устойчивому фронту коранга 1 в гладком $n$-мерном многообразии. Гиперповерхность $Г$ может иметь только две особенности коразмерности 4 , а именно особенности $4 A_{1}$ и $A_{3}+A_{1}$.

1) Множество $C_{\text {in }}\left(\left(4 A_{1}\right)_{\Gamma}\right)$ состоит из многообразий особенностей $4 A_{1}, A_{3}+2 A_{1}$, $2 A_{3}, A_{5}+A_{1}, A_{7}$ гиперповерхности $Г$. Множество $\partial_{\text {out }}\left(\left(4 A_{1}\right)_{\Gamma}\right)$ состоит из многообразий особенностей $5 A_{1}, 6 A_{1}, A_{3}+3 A_{1}, 7 A_{1}, A_{3}+4 A_{1}, 2 A_{3}+A_{1}, A_{5}+2 A_{1}$ и всех 
особенностей коразмерности больше 7. Поэтому для любого нечетного $n \geqslant 5$ из формулы (3) и теоремы 2.8 получаем следуюшее соотношение между эйлеровыми характеристиками многообразий особенностей гиперповерхности $\Gamma$ :

$$
\begin{aligned}
24 \chi\left(4 A_{1}\right)= & 60 \chi\left(5 A_{1}\right)+12 \chi\left(A_{3}+2 A_{1}\right)-180 \chi\left(6 A_{1}\right)-30 \chi\left(A_{3}+3 A_{1}\right) \\
& -6 \chi\left(2 A_{3}\right)-4 \chi\left(A_{5}+A_{1}\right)+420 \chi\left(7 A_{1}\right)+96 \chi\left(A_{3}+4 A_{1}\right) \\
& +15 \chi\left(2 A_{3}+A_{1}\right)+10 \chi\left(A_{5}+2 A_{1}\right)+\chi\left(A_{7}\right)+\cdots
\end{aligned}
$$

(здесь индекс Г опущен, а многоточие обозначает линейную комбинацию эйлеровых характеристик многообразий особенностей, коразмерность которых больше 7).

$2)$ Множество $C_{\text {in }}\left(\left(A_{3}+A_{1}\right)_{\Gamma}\right)$ состоит из многообразий особенностей $A_{3}+A_{1}$ и $A_{5}$ гиперповерхности $\Gamma$. Множество $\partial_{\text {out }}\left(\left(A_{3}+A_{1}\right)_{\Gamma}\right)$ состоит из многообразий особенностей $A_{3}+2 A_{1}$ и всех особенностей коразмерности больше 5 , кроме особенностей вида $m A_{1}$. Поэтому для любого нечетного $n \geqslant 5$ имеем

$$
\begin{aligned}
2 \chi\left(A_{3}+A_{1}\right)= & 2 \chi\left(A_{3}+2 A_{1}\right)+2 \chi\left(A_{5}\right)-3 \chi\left(A_{3}+3 A_{1}\right)-2 \chi\left(2 A_{3}\right) \\
& -2 \chi\left(A_{5}+A_{1}\right)+4 \chi\left(A_{3}+4 A_{1}\right)+4 \chi\left(2 A_{3}+A_{1}\right) \\
& +3 \chi\left(A_{5}+2 A_{1}\right)+\chi\left(A_{7}\right)+\cdots
\end{aligned}
$$

(многоточие обозначает то же самое, что и для особенности $4 A_{1}$ ).

ДОКАЗАТЕЛЬСТВО ТЕОРЕМЫ 1.9. Воспользуемся предложением 3.8 и напишем соотношение (3) для каждого класса особенностей гиперповерхности Г такого, что многообразие этих особенностей имеет нечетную размерность. В результате получится огромная система линейных однородных уравнений, неизвестньми которой являются эйлеровы характеристики всех многообразий особенностей рассматриваемой гиперповерхности.

Эта система легко представляется в треугольном виде относительно эйлеровых характеристик нечетномерных многообразий особенностей (необходимо лишь правильно занумеровать уравнения и неизвестные). Следовательно, эти эйлеровы характеристики линейно выражаются через остальные. Формулы, приведенные в пп. 2) и 3 ) теоремы 1.9 , получены путем решения указанной системы на компьютере с использованием пакета программ Mathematica.

\section{$\S 4$. Особенности остова коразмерности 1 канонической стратификации}

Рассмотрим (приводимую особую) гиперповерхность $\Sigma$ в гладком $n$-мерном многообразии $V$. Зафиксируем произвольное натуральное $\varkappa \leqslant n$ и любое целое неотрицательное $m \leqslant \varkappa+1$.

ОПРЕДЕЛЕНИЕ 4.1. Гиперповерхность $\Sigma$ имеет особенность $\mathscr{S}_{\varkappa}^{m}, m>0$, в точке $x$, если ее росток $(\Sigma, x)$ в этой точке диффеоморфен ростку в нуле объединения следующих гиперповерхностей в $\mathbb{R}^{n}=\left\{\left(\lambda_{1}, \ldots, \lambda_{n}\right)\right\}$ :

1) гиперповерхности $\mathscr{F}$, образованной точками, для которых функция

$$
S\left(t, \lambda_{m}, \ldots, \lambda_{\varkappa}\right)=t^{\varkappa-m+1}+\sum_{i=m}^{\varkappa} \lambda_{i} t^{i-m}
$$


как многочлен от $t$ имеет кратный вешественный корень (эта гиперповерхность не пуста только при $m<\varkappa$ );

2) $m$ гладких гиперповерхностей $H_{i}, i=1, \ldots, m$, заданных уравнениями

$$
H_{i}:\left.\quad S\right|_{t=-\left(\lambda_{i}+\cdots+\lambda_{m-1}\right)}=0, \quad i<m ; \quad H_{m}:\left.\quad S\right|_{t=0}=0
$$

(этот набор гиперповерхностей не пуст только при $m \leqslant \varkappa$ );

3) $m(m-1) / 2$ гиперплоскостей $\Pi_{i, j}, 1 \leqslant i \leqslant j \leqslant m$, заданных уравнениями

$$
\Pi_{i, j}: \quad \lambda_{i}+\cdots+\lambda_{j-1}=0
$$

(этот набор гиперповерхностей не пуст только при $m>1$ ).

Особенностями $\mathscr{S}_{\varkappa}^{0}$ әиперповерхности $\Sigma$ будем называть обычные ее особенности $A_{\varkappa}$.

Число $\varkappa$ называется показателем вырожденности особенности $\mathscr{S}_{\varkappa}^{m}$. Множество $\left(\mathscr{S}_{\varkappa}^{m}\right)_{\Sigma}$ особенностей $\mathscr{S}_{\varkappa}^{m}$ гиперповерхности $\Sigma$ является гладким подмногообразием коразмерности $\varkappa$ в объемлющем многообразии $V$ (вообще говоря, незамкнутым).

ЗАмЕчАниЕ 4.2. Напомним (см. [11]), что упорядоченная пара $(H, \Delta)$ гиперповерхностей $H$ и $\Delta$ в многообразии $V$ имеет особенность $B_{\mu}$ в точке $x \in H$, если росток $((H, \Delta), x)$ этой пары диффеоморфен ростку в $0 \in \mathbb{R}^{n}=\left\{\left(\lambda_{0}, \ldots, \lambda_{n-1}\right)\right\}$ пары, состоящей из гиперплоскости $\lambda_{0}=0$ и гиперповерхности, образованной точками, для которых многочлен

$$
t^{\mu}+\lambda_{\mu-1} t^{\mu-1}+\cdots+\lambda_{1} t+\lambda_{0}
$$

от $t$ имеет кратньй вешественный корень (при $\mu=1$ вторая компонента ростка пары пуста). Легко видеть, что каждая пара $\left(H_{i}, \mathscr{F}\right), i=1, \ldots, m$, имеет особенность $B_{\varkappa-m+1}$ в нуле.

Действительно,

$$
\begin{aligned}
\left.\frac{\partial^{p} S}{\partial\left(t+\lambda_{i}+\cdots+\lambda_{m-1}\right)^{p}}\right|_{t=\lambda_{i}=\cdots=\lambda_{m-1}=0} & =\left.\frac{\partial^{p} S}{\partial t^{p}}\right|_{t=\lambda_{i}=\cdots=\lambda_{m-1}=0} \\
& = \begin{cases}p !, & \text { если } p=\varkappa-m+1 ; \\
p ! \lambda_{m+p}, & \text { если } 0 \leqslant p \leqslant \varkappa-m .\end{cases}
\end{aligned}
$$

Поэтому росток в нуле функции $S$ как функции от $t$ является версальной деформацией ростка в нуле функции $\left(t+\lambda_{i}+\cdots+\lambda_{m-1}\right)^{\varkappa-m+1}$ на полупрямой с краем $t+\lambda_{i}+\cdots+\lambda_{m-1}=0$.

ЗАМЕЧАНИЕ 4.3. Для любых $1 \leqslant i<j \leqslant m$

$$
H_{i} \cap \Pi_{i, j}=H_{j} \cap \Pi_{i, j}
$$

Если $m<\varkappa$, то особенность $\mathscr{S}_{\varkappa}^{m}$ имеет $\left(m^{2}+m+2\right) / 2$ неприводимых компонент. 
ПримеР 4.4. Рассмотрим росток гиперповерхности с особенностью $\mathscr{S}_{3}^{2}$ в трехмерном пространстве. Этот росток диффеоморфен ростку в нуле объединения следующих четырех гладких поверхностей в $\mathbb{R}^{3}=\left\{\left(\lambda_{1}, \lambda_{2}, \lambda_{3}\right)\right\}$ :

1) дискриминанта $\lambda_{3}^{2}-4 \lambda_{2}=0$ квадратного трехчлена $t^{2}+\lambda_{3} t+\lambda_{2}$;

2) поверхности $\lambda_{1}^{2}-\lambda_{1} \lambda_{3}+\lambda_{2}=0$ и плоскости $\lambda_{2}=0$;

3) плоскости $\lambda_{1}=0$.

В координатах

$$
x=\lambda_{1}-\frac{\lambda_{3}}{2}, \quad y=-\frac{\lambda_{3}}{2}, \quad z=\frac{\lambda_{3}^{2}}{4}-\lambda_{2}
$$

эти поверхности задаются уравнениями (соответственно)

$$
z=0, \quad z=x^{2}, \quad z=y^{2}, \quad y=x .
$$

Их объединение изображено на рис. 3 (указанные на рисунке обозначения нужны для примера 4.8).

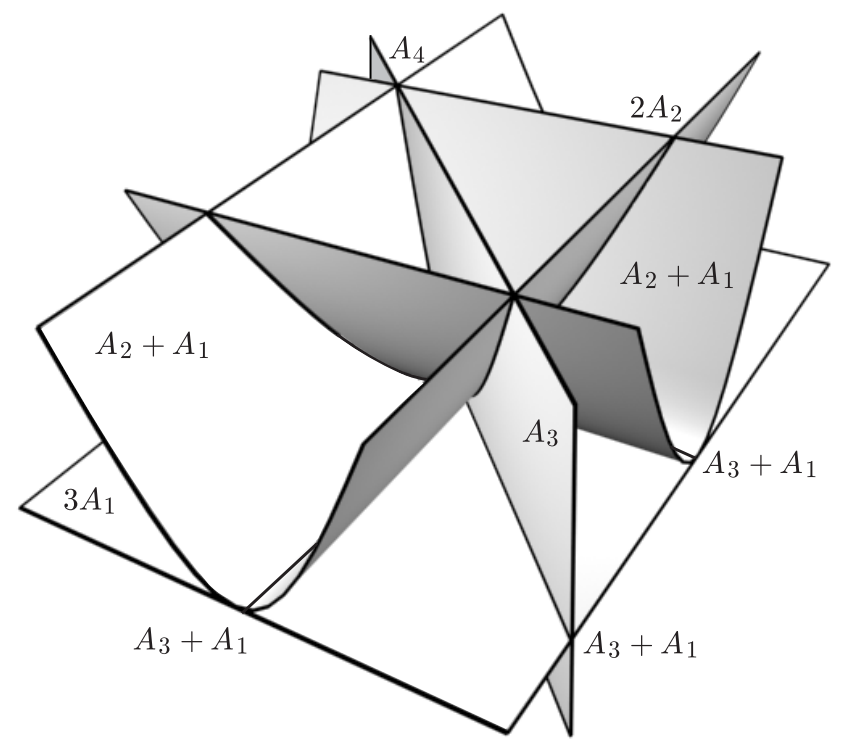

Рис. 3. Особенность $\mathscr{S}_{3}^{2}$

Рассмотрим свободную абелеву полугруппу по сложению с образующими $\mathscr{S}_{\varkappa}^{m}$, где $\varkappa \in \mathbb{N}, m \in \mathbb{Z}, 0 \leqslant m \leqslant \varkappa+1$.

ОПРЕДЕЛЕНИЕ 4.5. Гиперповерхность $\Sigma$ имеет особенность $\mathscr{S}_{\varkappa_{1}}^{m_{1}}+\cdots+\mathscr{S}_{\varkappa_{r}}^{m_{r}}$ в точке $x$, если ее росток $(\Sigma, x)$ является ростком объединения $r$ гиперповерхностей $\Sigma_{1}, \ldots, \Sigma_{r}$, которые имеют особенности $\mathscr{S}_{\varkappa_{1}}^{m_{1}}, \ldots, \mathscr{S}_{\varkappa_{r}}^{m_{r}}$ в точке $x$, причем ростки многообразий $\left(\mathscr{S}_{\varkappa_{1}}^{m_{1}}\right)_{\Sigma_{1}}, \ldots,\left(\mathscr{S}_{\varkappa_{r}}^{m_{r}}\right)_{\Sigma_{r}}$ пересекаются в этой точке трансверсально. 
Числа $\varkappa_{1}, \ldots, \varkappa_{r}$ называются показателями вырожсенности особенности $\mathscr{S}=\mathscr{S}_{\varkappa_{1}}^{m_{1}}+\cdots+\mathscr{S}_{\varkappa_{r}}^{m_{r}}$. Их сумма codim $\mathscr{S}=\varkappa_{1}+\cdots+\varkappa_{r}$ называется коразмерностью этой особенности.

Коразмерность особенности не превьшает размерности $n$ объемлющего многообразия $V$. Множество $\mathscr{S}_{\Sigma}$ особенностей $\mathscr{S}$ гиперповерхности $\Sigma$ с данным (неупорядоченным) набором показателей вырожденности является гладким подмногообразием в $V$, коразмерность которого равна коразмерности особенности.

ПрЕДЛОЖЕНИЕ 4.6. Если $\Sigma \subset V$ - замкнутая гиперповерхность с особенностями типа $\mathscr{S}_{\varkappa_{1}}^{m_{1}}+\cdots+\mathscr{S}_{\varkappa_{r}}^{m_{r}}$, то ее разбиение на связные компоненты многообразий этих особенностей является минимальной $C^{\infty}$-стратификацией Уитни.

ДоказАТЕльство. Указанное разбиение является, очевидно, $C^{\infty}$-стратификацией, причем минимальной. Эта стратификация удовлетворяет обоим условиям Уитни согласно следуюшему обшему факту из теории стратифицированных множеств.

Рассмотрим $C^{\infty}$-стратификацию замкнутого подмножества $Z$ в гладком $n$-мерном многообразии $V$. Предположим, что любая пара ее стратов $X, Y$ таких, что $Y$ лежит в замыкании $\bar{X}$ страта $X$, удовлетворяет следующему условию: для любой точки $y \in Y$ существуют гладкие локальные координаты на многообразии $V$, в которых росток пары $(\bar{X}, Y)$ в точке $y$ является ростком в нуле пары в $\mathbb{R}^{n}$, представляющей собой прямое произведение $\mathbb{R}^{k}$ на пару $(\Delta, 0)$, где $\Delta$ - замкнутое полуалгебраическое подмножество в $\mathbb{R}^{n-k}$, содержащее нуль 0 . Тогда эта стратификация является стратификацией Уитни (см. [12] или [13]).

Предложение 4.6 доказано.

Пусть $\mathscr{F}$ - устойчивый фронт коранга 1 в многообразии $V$. Зафиксируем произвольную особенность $\mathscr{A}=A_{\mu_{1}}+\cdots+A_{\mu_{m}}$ этого фронта и рассмотрим характеристическое многообразие $\Phi_{\mathscr{A}}$ и характеристическое отображение $\varphi_{\mathscr{A}}$ особенности $\mathscr{A}$.

Tеорема 4.7. Для любого $\mathscr{A}$ остов $\operatorname{St}_{1}\left(\Phi_{\mathscr{A}}\right)$ коразмерности 1 канонической стратификации многообразия $\Phi_{\mathscr{A}}$ имеет лииь особенности типа $\mathscr{S}_{\varkappa_{1}}^{m_{1}}+\cdots+$ $\mathscr{S}_{\varkappa_{r}}^{m_{r}}$.

А именно, пусть $\xi \in \mathrm{St}_{1}\left(\Phi_{\mathscr{A}}\right)$ и фронт $\mathscr{F}$ имеет особенность $X=A_{\nu_{1}}+$ $\cdots+A_{\nu_{p}}$ в точке $x=\varphi_{\mathscr{A}}(\xi)$. Тогда существуют отображсение

$$
\alpha: I=\{1, \ldots, m\} \rightarrow J=\{1, \ldots, p\}
$$

и разбиение $J=J_{-} \cup J_{0} \cup J_{+}$множества $J$ на попарно непересекающиеся подмножества $J_{-}, J_{0}, J_{+}$такие, что:

1) $J_{-} \cup J_{+}$непусто;

2) если $j \in J_{-}$, mo $\alpha^{-1}(j)$ nусто;

3) если $j \in J_{0}$, то множество $\alpha^{-1}(j)$ состоит из одного әлемента и $\nu_{j}=\mu_{\alpha-1(j)}$

4) если $j \in J_{+}$, то множество $\alpha^{-1}(j)$ состоит из $m_{j} \geqslant 1$ элементов и

$$
\varkappa_{j}=\nu_{j}-\sum_{i \in \alpha^{-1}(j)} \mu_{i} \geqslant \max \left\{1, m_{j}-1\right\} ;
$$


5) гиперповерхность $\operatorname{St}_{1}\left(\Phi_{\mathscr{A}}\right)$ имеет в точке $\xi$ особенность

$$
\mathscr{S}=\sum_{j \in J_{-}} A_{\nu_{j}}+\sum_{j \in J_{+}} \mathscr{S}_{\varkappa_{j}}^{m_{j}}
$$

(коразмерность особенности $\mathscr{S}$ равна $\operatorname{codim} X-\operatorname{codim} \mathscr{A})$.

Обратно, для любого отображения $\alpha: I \rightarrow J$ и любого разбиения $J=$ $J_{-} \cup J_{0} \cup J_{+}$множества $J$ на попарно непересекающиеся подмножества $J_{-}, J_{0}, J_{+}$, удовлетворяющие условиям 1$\left.)-4\right)$, существует точка $\widetilde{\xi} \in \varphi_{\mathscr{A}}^{-1}(x)$, в которой гиперповерхность $\operatorname{St}_{1}\left(\Phi_{\mathscr{A}}\right)$ имеет особенность $\mathscr{S}$.

Теорема 4.7 доказана в $\S 6$ (она является следствием предложения 6.4).

Пример 4.8. Пусть $\mathscr{F}$ - четырехмерный фронт с особенностью $A_{5}$ в точке $x$. Рассмотрим характеристическое многообразие $\Phi_{2 A_{1}}$ и характеристическое отображение $\varphi_{2 A_{1}}$ особенности $2 A_{1}$ этого фронта. Остов $\operatorname{St}_{1}\left(\Phi_{2 A_{1}}\right)$ коразмерности 1 канонической стратификации многообразия $\Phi_{2 A_{1}}$ имеет особенность $\mathscr{S}_{3}^{2}$ в точке $\xi=\varphi_{2 A_{1}}^{-1}(x)$. Росток $\left(\operatorname{St}_{1}\left(\Phi_{2 A_{1}}\right), \xi\right)$ изображен на рис. 3 в гладких координатах примера 4.4 .

Страты канонической стратификации многообразия $\Phi_{2 A_{1}}$, имеющие ненулевую коразмерность, обозначены классами особенностей фронта $\mathscr{F}$ в образах соответствуюших точек при отображении $\varphi_{2} A_{1}$. Не виден лишь 0 -мерный страт $A_{5}$, являющийся общей точкой всех четырех поверхностей. Этот страт примыкает к десяти одномерным стратам, среди которых два страта типа $A_{4}$, два страта $2 A_{2}$ и шесть стратов $A_{3}+A_{1}$ (геометрический смысл каждой из четырех изображенных поверхностей объяснен в примере 6.3$)$.

\section{§5. Вспомогательные утверждения}

В этом параграфе сформулированы некоторые простые алгебраические факты, необходимые для доказательства теоремы 4.7.

Лемма 5.1. Справедливо следующее:

$$
\sum_{i=0}^{l}(-1)^{i}\left(\begin{array}{c}
k+i \\
i
\end{array}\right)\left(\begin{array}{c}
n \\
l-i
\end{array}\right)=\left(\begin{array}{c}
n-k-1 \\
l
\end{array}\right)
$$

для любых челых неотрицательных $k, l, n$ таких, что $k+l<n$.

Доказательство заключается в сравнении коэффициентов при одинаковых степенях переменной $t$ в рядах $(1+t)^{n-k-1}$ и $(1+t)^{n}(1+t)^{-(k+1)}$.

ЛЕмма 5.2. Пусть

$$
P=t^{n}+q_{n-1} t^{n-1}+\cdots+q_{1} t+q_{0}
$$

- многочлен от $t$. Предполохим, что $t_{*}-$ корень многочлена $P-q_{0}$, причем его кратность равна $k$. Тогда либо $t_{*}=0$, либо $t_{*}-($ ненулевой $)$ корень уравнения

$$
\frac{P-q_{0}}{t}=0
$$

кратности $k$.

Это утверждение очевидно. 
Лемма 5.3. Пусть $P=t^{n}+q_{n-1} t^{n-1}+\cdots+q_{1} t+q_{0}-$ многочлен от $t u$

$$
P_{\mu}=t^{n-\mu}+\sum_{i=\mu}^{n-1} q_{i} t^{i-\mu}, \quad \mu=0,1, \ldots, n
$$

Тогда для любого $\mu<n$ многочлен

$$
R_{\mu}=v^{n-\mu-1}+\sum_{i=0}^{n-\mu-2} \frac{1}{(i+\mu+1) !} \frac{d^{i+\mu+1} P}{d t^{i+\mu+1}} v^{i}
$$

от $v$ можсно представить в виде

$$
R_{\mu}=\frac{1}{\mu !} \sum_{k=0}^{n-\mu-1} \frac{d^{\mu} P_{k+1}}{d t^{\mu}}(v+t)^{k}
$$

В частности, для любого $t_{*} \neq 0 u k=0,1, \ldots, n-\mu-1$

$$
\left.\frac{d^{\mu}}{d t^{\mu}}\left(\frac{P-\left(q_{k} t^{k}+\cdots+q_{1} t+q_{0}\right)}{t^{k+1}}\right)\right|_{t=t_{*}}=\left.\frac{\mu !}{k !} \frac{\partial^{k} R_{\mu}}{\partial v^{k}}\right|_{v=-t_{*}} .
$$

ДокАЗАТЕльство. Действительно,

$$
\begin{aligned}
\left.\frac{\partial^{k} R_{\mu}}{\partial v^{k}}\right|_{v=-t}= & \sum_{i=k}^{n-\mu-1} \frac{i !}{(i+\mu+1) !(i-k) !} \frac{d^{i+\mu+1} P}{d t^{i+\mu+1}}(-t)^{i-k} \\
= & k ! \sum_{i=k}^{n-\mu-1}(-1)^{i-k}\left(\begin{array}{c}
i \\
i-k
\end{array}\right)\left(\left(\begin{array}{c}
n \\
i+\mu+1
\end{array}\right) t^{n-\mu-k-1}\right. \\
& \left.+\sum_{r=i+\mu+1}^{n-1}\left(\begin{array}{c}
r \\
i+\mu+1
\end{array}\right) q_{r} t^{r-\mu-k-1}\right) \\
= & k !\left(t^{n-\mu-k-1} \sum_{i=0}^{n-\mu-k-1}(-1)^{i}\left(\begin{array}{c}
k+i \\
i
\end{array}\right)\left(\begin{array}{c}
n \\
i+\mu+k+1
\end{array}\right)\right. \\
& \left.+\sum_{r=\mu+k+1}^{n-1} q_{r} t^{r-\mu-k-1} \sum_{i=0}^{r-\mu-k-1}(-1)^{i}\left(\begin{array}{c}
k+i \\
i
\end{array}\right)\left(\begin{array}{c}
r \\
i+\mu+k+1
\end{array}\right)\right) .
\end{aligned}
$$

Отсюда по формуле (4) получаем

$$
\begin{aligned}
\left.\frac{\partial^{k} R_{\mu}}{\partial v^{k}}\right|_{v=-t} & =k !\left(\left(\begin{array}{c}
n-k-1 \\
\mu
\end{array}\right) t^{n-\mu-k-1}+\sum_{r=\mu+k+1}^{n-1}\left(\begin{array}{c}
r-k-1 \\
\mu
\end{array}\right) q_{r} t^{r-\mu-k-1}\right) \\
& =\frac{k !}{\mu !} \frac{d^{\mu} P_{k+1}}{d t^{\mu}} .
\end{aligned}
$$

Лемма 5.3 доказана. 
ЛЕмма 5.4. Пусть $t_{*}-$ ненулевой корень многочлена

$$
P=t^{n}+q_{n-1} t^{n-1}+\cdots+q_{1} t+q_{0}
$$

om t. Предположим, что кратность этого корня равна $\mu+1 u$

$$
\left.\frac{d^{\mu}}{d t^{\mu}}\left(\frac{P-\left(q_{k} t^{k}+\cdots+q_{1} t+q_{0}\right)}{t^{k+1}}\right)\right|_{t=t_{*}}=0, \quad k=0,1, \ldots, r
$$

для некоторого целого неотрицательного $r<n-\mu$. Тогда $q_{0}=\cdots=q_{r}=0$.

Действительно, указанные равенства определяют систему линейных однородных уравнений

$$
\left.\frac{d^{\mu}}{d t^{\mu}}\left(\frac{q_{k}}{t}+\cdots+\frac{q_{1}}{t^{k}}+\frac{q_{0}}{t^{k+1}}\right)\right|_{t=t_{*}}=0, \quad k=0,1, \ldots, r,
$$

с неизвестньми $q_{0}, \ldots, q_{r}$. Эта система имеет только тривиальное решение.

Лемма 5.5 [4]. Пусть $u, t \in \mathbb{R}, \bar{q}=\left(q_{2}, \ldots, q_{n-1}\right) \in \mathbb{R}^{n-2}$,

$$
\begin{aligned}
S(t, \bar{q}) & =t^{\nu+1}+q_{\nu-1} t^{\nu-1}+\cdots+q_{2} t^{2}, \\
F(u, t, \bar{q}) & =S(u, \bar{q})-S(t, \bar{q})-(u-t) \frac{\partial}{\partial t} S(t, \bar{q}) .
\end{aligned}
$$

Предположим, что корень $u=t$ многочлена $F$ (как многочлена от и) имеет кратность $\mu+1 \geqslant 2$. Тогда

$$
F(u, t, \bar{q})=(u-t)^{\mu+1} G_{\mu}\left(u, t, q_{\mu+1}, \ldots, q_{n-1}\right)
$$

əдe

$$
G_{\mu}\left(u, t, q_{\mu+1}, \ldots, q_{n-1}\right)=\sum_{i=\mu}^{\nu} \frac{1}{(i+1) !} \frac{\partial^{i+1} S(t, \bar{q})}{\partial t^{i+1}}(u-t)^{i-\mu}
$$

причем $\left.G_{\mu}\right|_{u=t} \neq 0$.

\section{§6. Доказательство теоремы 4.7}

В дальнейшем мы будем использовать координатное описание $A_{\mu}$-преобразования устойчивого фронта коранга 1.

ПРЕДЛОЖЕНИЕ 6.1. Пусть $t \in \mathbb{R}, \bar{q}=\left(q_{2}, \ldots, q_{n-1}\right) \in \mathbb{R}^{n-2}$,

$$
S(t, \bar{q})=t^{\nu+1}+q_{\nu-1} t^{\nu-1}+\cdots+q_{2} t^{2}
$$

и $\mathscr{F}$ - фронт лежсандрова отображсения

$$
\mathbb{R}^{n-1} \rightarrow \mathbb{R}^{n}, \quad(t, \bar{q}) \mapsto\left(-\frac{\partial}{\partial t} S(t, \bar{q}), \bar{q}, S(t, \bar{q})-t \frac{\partial}{\partial t} S(t, \bar{q})\right) .
$$

Тогда набор $\left(t, q_{\mu+1}, \ldots, q_{n-1}\right) \in \mathbb{R}^{n-\mu}$ определяет координаты на характеристическом многообразии $\Phi_{A_{\mu}}$ особенности $A_{\mu}$ фронта $\mathscr{F}$. В этих координатах фронт $\left[A_{\mu}\right](\mathscr{F}) \subset \Phi_{A_{\mu}}$ является множсеством точек, для которых функиия

$$
\sum_{i=\mu}^{\nu} \frac{1}{(i+1) !} \frac{\partial^{i+1} S(t, \bar{q})}{\partial t^{i+1}}(u-t)^{i-\mu}
$$

как многочлен от $u-t$ имеет кратный вещественный корень. 
ДокАЗАТЕЛЬСтво. Подмногообразие $\Phi_{A_{\mu}} \subseteq \mathbb{R}^{n-1}$ задается уравнениями

$$
\frac{\partial^{2} S}{\partial t^{2}}=\frac{\partial^{3} S}{\partial t^{3}}=\cdots=\frac{\partial^{\mu} S}{\partial t^{\mu}}=0
$$

$\Phi$ ронт $\left[A_{\mu}\right](\mathscr{F})$ является замыканием множества точек $(t, \bar{q}) \in \Phi_{A_{\mu}}$, для которых функция

$$
F(u, t, \bar{q})=S(u, \bar{q})-S(t, \bar{q})-(u-t) \frac{\partial}{\partial t} S(t, \bar{q})
$$

как многочлен от $u$ имеет кратный вешественный корень $u \neq t$. Поэтому предложение 6.1 следует из леммы 5.5 .

Пусть $\mathscr{F}$ - фронт устойчивого лежандрова отображения $f: L \rightarrow V$ коранга 1 в гладком $n$-мерном многообразии $V$. Зафиксируем особенность $\mathscr{A}=A_{\mu_{1}}+$ $\cdots+A_{\mu_{m}}$ этого фронта.

Рассмотрим характеристическое многообразие $\Phi_{\mathscr{A}}$ и характеристическое отображение $\varphi_{\mathscr{A}}$ особенности $\mathscr{A}$. Выберем произвольную точку $\xi_{m}$ остова $\operatorname{St}_{1}\left(\Phi_{\mathscr{A}}\right)$ коразмерности 1 канонической стратификации многообразия $\Phi_{\mathscr{A}}$.

ПРЕДЛОЖЕНИЕ 6.2. Пусть фронт $\mathscr{F}$ имеет особенность $A_{\nu}$ в точке $x=$ $\varphi_{\mathscr{A}}\left(\xi_{m}\right)$. Тогда $n_{m}=\nu-\operatorname{codim} \mathscr{A} \geqslant \max \{1, m-1\}$ и гиперповерхность $\operatorname{St}_{1}\left(\Phi_{\mathscr{A}}\right)$ имеет особенность $\mathscr{S}_{n_{m}}^{m}$ в точке $\xi_{m}$. Более того, существуют локальные координать

$$
\left(t, Q_{0}^{m}, \ldots, Q_{n_{m}-m-1}^{m}, y_{3}, \ldots, y_{\operatorname{deg} \mathscr{A}}, z_{1}, \ldots, z_{n-\nu}\right)
$$

на многообразии $L$ в окрестности точки $\xi_{1}=f^{-1}(x)$ и локальные координа$m b l$

$$
\left(t_{1}, \ldots, t_{m-1}, Q_{0}^{m}, \ldots, Q_{n_{m}-m-1}^{m}, Q_{n_{m}-m}^{m}, z_{1}, \ldots, z_{n-\nu}\right)
$$

на многообразии $\Phi_{\mathscr{A}}$ в окрестности точки $\xi_{m}$ такие, что справедливы следующие утверждения.

1) Для любих $t_{1}, \ldots, t_{m-1}, Q_{0}^{m}, \ldots, Q_{n_{m}-m}^{m}, z_{1}, \ldots, z_{n-\nu}$, достаточно близких к нулю, точки

$$
\begin{gathered}
\left(t_{0}+\cdots+t_{i}, Q_{0}^{m}, \ldots, Q_{n_{m}-m-1}^{m}, y_{3}, \ldots, y_{\operatorname{deg} \mathscr{A}}, z_{1}, \ldots, z_{n-\nu}\right) \in L \\
i=0, \ldots, m-1
\end{gathered}
$$

əде

$$
\sum_{i=1}^{m-1}\left(\nu-\mu_{1}-\cdots-\mu_{i}-i+1\right) t_{i}+(\nu+1) t_{0}=\left\{\begin{array}{lll}
Q_{n_{m}-m}^{m}, & \text { если } n_{m} \geqslant m \\
0, & \text { если } n_{m}<m
\end{array}\right.
$$

а $y_{3}, \ldots, y_{\mathrm{deg} \mathscr{A}}$ гладко зависят от $t_{1}, \ldots, t_{m-1}, Q_{0}^{m}, \ldots, Q_{n_{m}-m}^{m}$, входят в полный прообраз точки

$$
\varphi_{\mathscr{A}}\left(t_{1}, \ldots, t_{m-1}, Q_{0}^{m}, \ldots, Q_{n_{m}-m}^{m}, z_{1}, \ldots, z_{n-\nu}\right) \in V
$$

относительно отображения $f$. 
2) Росток $\left(\operatorname{St}_{1}\left(\Phi_{\mathscr{A}}\right), \xi_{m}\right)$ является ростком в нуле обвединения следующих гиперповерхностей:

а) гиперповерхности $\mathscr{F}^{m}$, образованной точками, для которых функиия

$$
S_{m}\left(t_{m}, Q_{0}^{m}, \ldots, Q_{n_{m}-m}^{m}\right)=t_{m}^{n_{m}-m+1}+\sum_{i=0}^{n_{m}-m} Q_{i}^{m} t_{m}^{i}
$$

как многочлен от $t_{m}$ имеет кратный вещественный корень (әиперповерхность $\mathscr{F}^{m}$ не пуста только при $n_{m}>m$ );

b) $m$ гладких гиперповерхностей $H_{i}^{m}$, заданных уравнениями

$$
\left.S_{m}\right|_{t_{i}+\cdots+t_{m}=0}=0, \quad i=1, \ldots, m
$$

(этот набор гиперповерхностей не пуст только при $n_{m}>m-1$ );

c) $m(m-1) / 2$ әиперплоскостей $\Pi_{i, j}^{m}$, заданных уравнениями

$$
t_{i}+\cdots+t_{j-1}=0, \quad 1 \leqslant i<j \leqslant m
$$

( этот набор гиперповерхностей не пуст только при $m>1$ ).

3) Ростки гиперповерхностей $\mathscr{F}^{m}, H_{i}^{m}, \Pi_{i, j}^{m}$ в нуле являются полными прообразами ростков множсеств

$$
{\overline{\left(\mathscr{A}+A_{1}\right)_{\mathscr{F}}}}, \quad{\overline{\left(\mathscr{A}-A_{\mu_{i}}+A_{\mu_{i}+1}\right)_{\mathscr{F}}}}, \quad{\overline{\left(\mathscr{A}-A_{\mu_{i}}-A_{\mu_{j}}+A_{\mu_{i}+\mu_{j}+1}\right)_{\mathscr{F}}}}
$$

в точке $x$ соответственно относительно ростка отобрахсения ке $\xi_{m}$. При этом росток гиперповерхности $\mathscr{F}^{m}$ является ростком фронта $[\mathscr{A}](\mathscr{F}) \subset \Phi_{\mathscr{A}}$.

ДокАЗАТЕльство. Утверждение доказьвается индукцией по $m$. Сначала рассмотрим подробно случаи $m=1$ и $m=2$.

Случай $m=1$. В подходящих локальных координатах на многообразиях $L$ и $V$ росток отображения $f$ в точке $\xi_{1}$ задается формулой

$$
\begin{gathered}
\left(f, \xi_{1}\right):\left(\mathbb{R}^{n-1}, 0\right) \rightarrow\left(\mathbb{R}^{n}, 0\right) \\
\left(t_{0}, \bar{q}, \bar{z}\right) \mapsto\left(-\frac{\partial}{\partial t_{0}} S\left(t_{0}, \bar{q}\right), S\left(t_{0}, \bar{q}\right)-t_{0} \frac{\partial}{\partial t_{0}} S\left(t_{0}, \bar{q}\right), \bar{q}, \bar{z}\right),
\end{gathered}
$$

где

$$
\begin{gathered}
\bar{q}=\left(q_{2}, \ldots, q_{\nu-1}\right), \quad \bar{z}=\left(z_{1}, \ldots, z_{n-\nu}\right) \\
S\left(t_{0}, \bar{q}\right)=t_{0}^{\nu+1}+q_{\nu-1} t_{0}^{\nu-1}+\cdots+q_{2} t_{0}^{2}
\end{gathered}
$$

(см. [1]). Росток многообразия $\Phi_{A_{1}} \subseteq L$ в этой точке задается уравнениями

$$
\frac{\partial^{2} S}{\partial t_{0}^{2}}=\frac{\partial^{3} S}{\partial t_{0}^{3}}=\cdots=\frac{\partial^{\mu_{1}} S}{\partial t_{0}^{\mu_{1}}}=0
$$


Набор $\left(Q_{0}^{1}, \ldots, Q_{n_{1}-1}^{1}, \bar{z}\right) \in \mathbb{R}^{n-\mu_{1}}$, где $n_{1}=\nu-\mu_{1}$ и

$$
Q_{i}^{1}=\frac{1}{\left(i+\mu_{1}+1\right) !} \frac{\partial^{i+\mu_{1}+1} S}{\partial t_{0}^{i+\mu_{1}+1}}, \quad i=0, \ldots, n_{1}-1
$$

определяет локальные координаты на $\Phi_{A_{\mu_{1}}}$ (заметим, что $\left.Q_{n_{1}-1}^{1}=(\nu+1) t_{0}\right)$.

В этих координатах росток гиперповерхности $\Phi_{A_{\mu_{1}+1}} \subset \Phi_{A_{\mu_{1}}}$ в точке $\xi_{1}$ задается уравнением

$$
H_{1}^{1}: \quad Q_{0}^{1}=0
$$

Росток фронта $\mathscr{F}^{1}=\left[A_{\mu_{1}}\right](\mathscr{F}) \subset \Phi_{A_{\mu_{1}}}$ является ростком в нуле множества точек, для которых функция

$$
S_{1}\left(t_{1}, Q_{0}^{1}, \ldots, Q_{n_{1}-1}^{1}\right)=t_{1}^{n_{1}}+\sum_{i=0}^{n_{1}-1} Q_{i}^{1} t_{1}^{i}
$$

как многочлен от $t_{1}$ имеет кратный вещественньй корень (см. предложение 6.1; для соответствующих значений $Q_{0}^{1}, \ldots, Q_{n_{1}-1}^{1}$ точка $\left(t_{0}+t_{1}, \bar{q}, \bar{z}\right) \in L$, где $\bar{q}$ гладко зависит от $Q_{0}^{1}, \ldots, Q_{n_{1}-1}^{1}$, входит в полный прообраз точки $\varphi_{A_{\mu_{1}}}\left(Q_{0}^{1}, \ldots, Q_{n_{1}-1}^{1}, \bar{z}\right)$ относительно отображения $f$ ).

По определению ростки гиперповерхностей $\mathscr{F}^{1}$ и $H_{1}^{1}$ в нуле являются полными

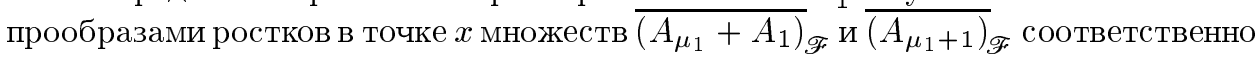
относительно ростка отображения $\varphi_{A_{\mu_{1}}}$ в точке $\xi_{1}$.

Случай $m=2$. Начиная с этого момента будем считать, что $\nu=n$. Из предыдущих формул видно, что это не ограничивает общности наших рассуждений.

Пусть далее $\Phi_{A_{\mu}}=\Phi_{A_{\mu}}^{1}$ и $\varphi_{A_{\mu}}=\varphi_{A_{\mu}}^{1}$. Через $\Phi_{A_{\mu}}^{2}$ и $\varphi_{A_{\mu}}^{2}$ обозначим характеристическое многообразие и характеристическое отображение особенности $A_{\mu}$ фронта $\mathscr{F}^{1}$ соответственно.

Очевидно, что набор $\left(t_{1}, Q_{2}^{1}, \ldots, Q_{n_{1}-1}^{1}\right) \in \mathbb{R}^{n_{1}-1}$ определяет локальные координаты на многообразии $\Phi_{A_{1}}^{2}$ в окрестности точки $\xi_{2}=\left(\varphi_{A_{1}}^{2}\right)^{-1}\left(\xi_{1}\right)$. Росток под-

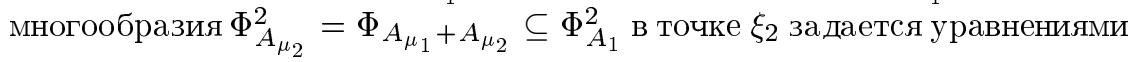

$$
\frac{\partial^{2} S_{1}}{\partial t_{1}^{2}}=\frac{\partial^{3} S_{1}}{\partial t_{1}^{3}}=\cdots=\frac{\partial^{\mu_{2}} S_{1}}{\partial t_{1}^{\mu_{2}}}=0
$$

Набор $\left(t_{1}, Q_{0}^{2}, \ldots, Q_{n_{2}-2}^{2}\right) \in \mathbb{R}^{n_{2}}$, где $n_{2}=n_{1}-\mu_{2}$ и

$$
Q_{i}^{2}=\frac{1}{\left(i+\mu_{2}+1\right) !} \frac{\partial^{i+\mu_{2}+1} S_{1}}{\partial t_{1}^{i+\mu_{2}+1}}, \quad i=0, \ldots, n_{2}-2
$$

служит локальнњми координатами на $\Phi_{A_{\mu_{2}}}^{2}$ (заметим, что $Q_{n_{2}-2}^{2}=n_{1} t_{1}+Q_{n_{1}-1}^{1}$ ).

В этих координатах росток гиперповерхности $\Phi_{A_{\mu_{2}+1}}^{2} \subset \Phi_{A_{\mu_{2}}}^{2}$ в точке $\xi_{2}$ задается уравнением

$$
H_{2}^{2}: \quad Q_{0}^{2}=0
$$


Росток фронта $\mathscr{F}^{2}=\left[A_{\mu_{2}}\right]\left(\mathscr{F}^{1}\right) \subset \Phi_{A_{\mu_{2}}}^{2}$ является ростком в нуле множества точек, для которых функция

$$
S_{2}\left(t_{2}, Q_{0}^{2}, \ldots, Q_{n_{2}-2}^{2}\right)=t_{2}^{n_{2}-1}+\sum_{i=0}^{n_{2}-2} Q_{i}^{2} t_{2}^{i}
$$

как многочлен от $t_{2}$ имеет кратный вещественньй корень (см. предложение 6.1; для соответствуюших $Q_{0}^{2}, \ldots, Q_{n_{2}-2}^{2}$ точка $\left(t_{0}+t_{1}+t_{2}, \bar{q}\right) \in L$, где $\bar{q}$ гладко зависит от $t_{1}, Q_{0}^{2}, \ldots, Q_{n_{2}-2}^{2}$, входит в полный прообраз точки $\varphi_{A_{\mu_{1}}}+A_{\mu_{2}}\left(t_{1}, Q_{0}^{2}, \ldots, Q_{n_{2}-2}^{2}\right)$ относительно отображения $f$ ).

Теперь рассмотрим пересечение гиперповерхностей $\mathscr{F}^{1}$ и $H_{1}^{1}$ в многообразии $\Phi_{A_{\mu_{1}}}^{1}$. Оно состоит из двух компонент: замыкания $H_{1}^{\text {tg }}$ множества точек касания и замькания $H_{1}^{\mathrm{tr}}$ множества точек трансверсального пересечения (гиперповерхности $H_{1}^{1}$ с фронтом $\mathscr{F}^{1}$ в гладких точках последнего).

Согласно лемме 5.2 росток в $\xi_{2}$ полного прообраза гиперповерхности $H_{1}^{\mathrm{tg}}$ относительно отображения $\varphi_{A_{\mu_{2}}}^{2}$ является ростком гладкой гиперповерхности в многообразии $\Phi_{A_{\mu_{2}}}^{2}$. В выбранных локальных координатах на $\Phi_{A_{\mu_{2}}}^{2}$ эта гиперповерхность задается уравнением

$$
\Pi_{1,2}^{2}: \quad t_{1}=0
$$

Росток полного прообраза гиперповерхности $H_{1}^{\mathrm{tr}}$ является ростком гладкой гиперповерхности, которая совпадает с замыканием гиперповерхности

$$
\frac{\partial^{\mu_{2}}}{\partial t_{1}^{\mu_{2}}}\left(\frac{S_{1}-Q_{0}^{1}}{t_{1}}\right)=0
$$

По лемме 5.3 (формула (5) при $k=0$ и $\mu=\mu_{2}$ ) эта гиперповерхность задается уравнением

$$
H_{1}^{2}:\left.\quad S_{2}\right|_{t_{2}=-t_{1}}=0
$$

Остается описать образы ростков гиперповерхностей $\mathscr{F}^{2}, H_{1}^{2}, H_{2}^{2}$ и $\Pi_{1,2}^{2}$ в нуле при отображении $\varphi_{\mu_{1}}+A_{\mu_{2}}$.

Образы ростков $\mathscr{F}^{2}$ и $H_{2}^{2}$ являются ростками в $x$ множеств $\overline{\left(A_{\mu_{1}}+A_{\mu_{2}}+A_{1}\right)}$

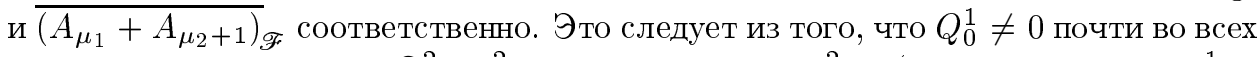
точках образов ростков $\mathscr{F}^{2}, H_{2}^{2}$ при отображении $\varphi_{A_{\mu_{2}}}^{2}$ (т.е. отображение $\varphi_{A_{\mu_{1}}}^{1}$ является иммерсией в этих точках).

Образ ростка $H_{1}^{2}$ является ростком множества $\overline{\left(A_{\mu_{1}+1}+A_{\mu_{2}}\right)_{\mathscr{F}}}$ в точке $x$, так как образ ростка $H_{1}^{2}$ при отображении $\varphi_{A_{\mu_{2}}}^{2}$ задается уравнением $Q_{0}^{1}=0$, причем $Q_{1}^{1} \neq 0$ почти всюду на этом образе. Наконец, образ ростка $\Pi_{1,2}^{2}$ является ростком множества ${\overline{\left(A_{\mu_{1}+\mu_{2}+1}\right)}}_{\mathscr{F}}$ в точке $x$, так как образ ростка $\Pi_{1,2}^{2}$ при отображении $\varphi_{A_{\mu_{2}}}^{2}$ задается уравнениями $Q_{0}^{1}=\cdots=Q_{\mu_{2}}^{1}=0$, причем $Q_{\mu_{2}+1}^{1} \neq 0$ почти во всех его точках. 
ПримеР 6.3. Рассмотрим фронт $\mathscr{F}$ из примера 4.8. Росток гиперповерхности $\operatorname{St}_{1}\left(\Phi_{\mathscr{A}}\right)$ в точке $\xi=\xi_{2}$ при $\mathscr{A}=2 A_{1}$ изображен на рис. 3 . Фронт $\mathscr{F}^{2}$ представлен здесь горизонтальной плоскостью. Гиперповерхности $H_{1}^{2}$ и $H_{2}^{2}$ представлены параболическими цилиндрами. Гиперплоскость $\Pi_{1,2}^{2}$ изображена вертикальной плоскостью.

Шаг индукции. Предположим, что мы выполнили (последовательно) $A_{\mu_{i}}$-преобразования фронтов $\mathscr{F}^{i-1}, i=1, \ldots, m_{0}-1, \mathscr{F}^{0}=\mathscr{F}$, и доказали предложение 6.2 для всех $m<m_{0}$. Выполним теперь $A_{\mu_{m_{0}}}$-преобразование фронта $\mathscr{F} m_{0}-1$ и докажем утверждение для $m=m_{0}$. Как и выше, мы будем считать, что $\nu=n$, и (для упрощения обозначений) опустим индекс 0 у числа $m_{0}$.

Рассмотрим характеристическое многообразие $\Phi_{A_{\mu}}^{m}$ и характеристическое отображение $\varphi_{A_{\mu}}^{m}$ особенности $A_{\mu}$ фронта $\mathscr{F}^{m-1}$. Очевидно, что набор

$$
\left(t_{1}, \ldots, t_{m-1}, Q_{2}^{m-1}, \ldots, Q_{n_{m-1}-m+1}^{m-1}\right) \in \mathbb{R}^{n_{m-1}-1}
$$

определяет локальные координаты на многообразии $\Phi_{A_{1}}^{m}$ в окрестности точки $\xi_{m}=$ $\left(\varphi_{A_{1}}^{m}\right)^{-1}\left(\xi_{m-1}\right)$.

Росток подмногообразия $\Phi_{A_{\mu m}}^{m}=\Phi_{\mathscr{A}} \subseteq \Phi_{A_{1}}^{m}$ в точке $\xi_{m}$ задается уравнениями

$$
\frac{\partial^{2} S_{m-1}}{\partial t_{m-1}^{2}}=\frac{\partial^{3} S_{m-1}}{\partial t_{m-1}^{3}}=\cdots=\frac{\partial^{\mu_{m}} S_{m-1}}{\partial t_{m-1}^{\mu_{m}}}=0 .
$$

Набор $\left(t_{1}, \ldots, t_{m-1}, Q_{0}^{m}, \ldots, Q_{n_{m}-m}^{m}\right) \in \mathbb{R}^{n_{m}}$, где $n_{m}=n_{m-1}-\mu_{m}$ и

$$
Q_{i}^{m}=\frac{1}{\left(i+\mu_{m}+1\right) !} \frac{\partial^{i+\mu_{m}+1} S_{m-1}}{\partial t_{m-1}^{i+\mu_{m}+1}}, \quad i=0, \ldots, n_{m}-m
$$

служит локальными координатами на $\Phi_{A_{m}}^{m}$ (заметим, что $Q_{n_{m}-m}^{m}=\left(n_{m-1}-\right.$ $\left.m+2) t_{m-1}+Q_{n_{m-1}-m+1}^{m-1}\right)$.

В этих координатах росток гиперповерхности $\Phi_{A_{\mu m+1}}^{m} \subset \Phi_{A_{\mu m}}^{m}$ в точке $\xi_{m}$ задается уравнением

$$
H_{m}^{m}: \quad Q_{0}^{m}=0
$$

Росток фронта $\mathscr{F}^{m}=\left[A_{\mu_{m}}\right]\left(\mathscr{F}^{m-1}\right) \subset \Phi_{A_{\mu m}}^{m}$ является ростком в нуле множества точек, для которых функция

$$
S_{m}\left(t_{m}, Q_{0}^{m}, \ldots, Q_{n_{m}-m}^{m}\right)=t_{m}^{n_{m}-m+1}+\sum_{i=0}^{n_{m}-m} Q_{i}^{m} t_{m}^{i}
$$

как многочлен от $t_{m}$ имеет кратный вещественный корень (см. предложение 6.1 ; для соответствующих значений $Q_{0}^{m}, \ldots, Q_{n_{m}-m}^{m}$ точка $\left(t_{0}+\cdots+t_{m}, \bar{q}\right) \in L$, где $\bar{q}$ гладко зависит от $t_{1}, \ldots, t_{m-1}, Q_{0}^{m}, \ldots, Q_{n_{m}-m}^{m_{m}}$, входит в полный прообраз точки

$$
\varphi_{\mathscr{A}}\left(t_{1}, \ldots, t_{m-1}, Q_{0}^{m}, \ldots, Q_{n_{m}-m}^{m}\right)
$$

относительно отображения $f)$. 
Теперь рассмотрим пересечения фронта $\mathscr{F}^{m-1}$ с гиперповерхностями $H_{i}^{m-1}, i=$ $1, \ldots, m-1$, и $\Pi_{i, j}^{m-1}, 1 \leqslant i<j \leqslant m-1$. Ростки в точке $\xi_{m}$ полных прообразов пересечений $\Pi_{i, j}^{m-1} \cap \mathscr{F}{ }^{m-1}$ относительно отображения $\varphi_{A_{\mu m}^{m}}^{m}$ являются ростками в нуле гиперплоскостей

$$
\Pi_{i, j}^{m}: \quad t_{i}+\cdots+t_{j-1}=0
$$

соответственно. Каждое пересечение $H_{i}^{m-1} \cap \mathscr{F}^{m-1}$ состоит из двух компонент: замыкания $H_{i}^{\mathrm{tg}}$ множества точек касания и замыкания $H_{i}^{\mathrm{tr}}$ множества точек трансверсального пересечения (гиперповерхности $H_{i}^{m-1}$ с фронтом $\mathscr{F}^{m-1}$ в гладких точках последнего).

Согласно лемме 5.2 росток в точке $\xi_{m}$ полного прообразагиперповерхности $H_{i}^{\mathrm{tg}}$ относительно отображения $\varphi_{A_{\mu m}}^{m}$ является ростком в нуле гиперплоскости

$$
\Pi_{i, m}^{m}: t_{i}+\cdots+t_{m-1}=0 .
$$

Росток полного прообраза гиперповерхности $H_{i}^{\text {tr }}$ является росткомгладкой гиперповерхности, которая совпадает с замыканием гиперповерхности

$$
\frac{\partial^{\mu_{m}}}{\partial\left(t_{i}+\cdots+t_{m-1}\right)^{\mu_{m}}}\left(\frac{S_{m-1}-\left.S_{m-1}\right|_{t_{i}+\cdots+t_{m-1}=0}}{t_{i}+\cdots+t_{m-1}}\right)=0 .
$$

По лемме 5.3 (формула (5) при $k=0$ и $\mu=\mu_{m}$ ) эта гиперповерхность задается уравнением

$$
H_{i}^{m}:\left.\quad S_{m}\right|_{t_{i}+\cdots+t_{m}=0}=0 .
$$

Остается описать образы ростков гиперповерхностей $\mathscr{F}^{m}, H_{i}^{m}, i=1, \ldots, m$, и $\Pi_{i, j}^{m}, 1 \leqslant i<j \leqslant m$, в нуле при отображении $\varphi_{\mathscr{A}}$. Для простоты мы будем обозначать их через $\varphi_{\mathscr{A}}\left(\mathscr{F}^{m}\right), \varphi_{\mathscr{A}}\left(H_{i}^{m}\right)$ и $\varphi_{\mathscr{A}}\left(\Pi_{i, j}^{m}\right)$ соответственно.

Пусть $\phi_{l}=\varphi_{A_{\mu_{l+1}}}^{l+1} \circ \cdots \circ \varphi_{A_{\mu m}}^{m}$, где $l=1, \ldots, m-1$. Рассмотрим образы $\phi_{l}\left(\mathscr{F}^{m}\right), \phi_{l}\left(H_{i}^{m}\right), \phi_{l}\left(\Pi_{i, j}^{m}\right)$ ростков гиперповерхностей $\mathscr{F}^{m}, H_{i}^{m}, \Pi_{i, j}^{m}$ в нуле относительно отображения $\phi_{l}$.

Легко видеть, что $Q_{0}^{l} \neq 0$ для любого $l$ почти во всех точках ростка $\phi_{l}\left(\mathscr{F}^{m}\right)$ (т.е. отображение $\varphi_{A_{\mu l}}^{l}$ является иммерсией в этих точках). Следовательно, росток $\varphi_{\mathscr{A}}\left(\mathscr{F}^{m}\right)$ является ростком множества $\overline{\left(\mathscr{A}+A_{1}\right)_{\mathscr{F}}}$ в точке $x$.

Росток $\varphi_{\mathscr{A}}\left(H_{i}^{m}\right)$ является ростком (в точке $x$ ) множества

$$
{\overline{\left(\mathscr{A}-A_{\mu_{i}}+A_{\mu_{i}+1}\right)_{\mathscr{F}}}} .
$$

Это следует из того, что $Q_{0}^{l} \neq 0$ для любого $l \neq i$ и $Q_{0}^{l}=0, Q_{1}^{l} \neq 0$ при $l=i$ почти всюду на $\phi_{l}\left(H_{i}^{m}\right)$.

Наконец, росток $\varphi_{\mathscr{A}}\left(\Pi_{i, j}^{m}\right)$ является ростком множества

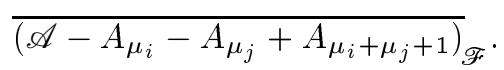

Это вытекает из того, что почти всюду на $\phi_{l}\left(\Pi_{i, j}^{m}\right)$ справедливо следуюшее:

1) $Q_{0}^{l} \neq 0$ для любого $l=j, \ldots, m-1$;

2) число $t_{l}=-\left(t_{i}+\cdots+t_{l-1}\right)$ является ненулевым корнем кратности $\mu_{j}+1$ многочлена $S_{l}$ при $l=i+1, \ldots, j-1$; в частности $Q_{0}^{l} \neq 0$;

3) $Q_{0}^{l}=\cdots=Q_{\mu_{j}}^{l}=0, Q_{\mu_{j}+1}^{l} \neq 0$ при $l=i$;

4) $Q_{0}^{l} \neq 0$ для любого $l=1, \ldots, i-1$. 
Свойства 1)-4) проверяются последовательно.

Свойство 1) очевидно.

Свойство 2) доказывается индукцией по убываюшему $l$. А именно, при $l=j-1$ свойство 2) справедливо по определению. Предположим, что число $t_{l}=-\left(t_{i}+\right.$ $\left.\cdots+t_{l-1}\right)$ является ненулевым корнем кратности $\mu_{j}+1$ многочлена $S_{l}$. Тогда по леммам 5.3 и 5.4 (при $P=S_{l-1}, \mu=\mu_{l}, R_{\mu}=S_{l}, v=t_{l}, t_{*}=t_{i}+\cdots+$ $\left.t_{l-1}, r=\mu_{j}\right)$ число $t_{l-1}=-\left(t_{i}+\cdots+t_{l-2}\right)$ является корнем кратности $\mu_{j}+1$ многочлена $S_{l-1}$. Если $l>i+1$, то этот корень не равен нулю почти во всех точках ростка $\phi_{l-1}\left(\Pi_{i, j}^{m}\right)$.

Свойство 3 ) следует из свойства 2) при $l=i+1$ и лемм 5.3 и 5.4 (при $P=S_{i}$, $\left.\mu=\mu_{i+1}, R_{\mu}=S_{i+1}, v=t_{i+1}, t_{*}=t_{i}, r=\mu_{j}\right)$.

Из свойства 3) немедленно следует свойство 4).

Таким образом, предложение 6.2 полностью доказано.

ПРЕДЛОЖЕНИЕ 6.4. Пусть фронт $\mathscr{F}$ имеет особенность $X=A_{\nu_{1}}+\cdots+A_{\nu_{p}}$ в точке $x=\varphi_{\mathscr{A}}\left(\xi_{m}\right)$. Рассмотрим неприводимье компоненты $\mathscr{F}_{1}, \ldots, \mathscr{F}_{p}$ ростка $(\mathscr{F}, x)$ и предположим, что әти компоненты имеют в точке $x$ особенности $A_{\nu_{1}}, \ldots, A_{\nu_{p}}$ соответственно. Тогда найдется разбиение

$$
I=\bigcup_{j=1}^{p} I_{j}
$$

множества $I=\{1, \ldots, m\}$ на попарно непересекающиеся подмножества $I_{j}$ такие, что справедливы следующие утверждения.

1) Для любого $j=1, \ldots, p$ множсество $I_{j}$ состоит из $m_{j} \geqslant 0$ чисел, упорядоченных по возрастанию, причем

$$
n_{j}=\nu_{j}-\sum_{i \in I_{j}} \mu_{i} \geqslant m_{j}-1, \quad \sum_{j=1}^{p} n_{j}=\operatorname{codim} X-\operatorname{codim} \mathscr{A} \geqslant 1 .
$$

2) Существуют локальнье координаты

$$
\left(\lambda_{1}^{1}, \ldots, \lambda_{n_{1}}^{1}, \ldots, \lambda_{1}^{p}, \ldots, \lambda_{n_{p}}^{p}, z_{1}, \ldots, z_{n-\operatorname{codim} X}\right)
$$

на многообразии $\Phi_{\mathscr{A}}$ такие, ито в этих координатах росток $\left(\operatorname{St}_{1}\left(\Phi_{\mathscr{A}}\right), \xi_{m}\right)$ является ростком в нуле обвединения $\bigcup_{j=1}^{p} \Sigma_{j}$ гиперповерхностей $\Sigma_{j}$, которье определяются следующим образом:

- если $m_{j}=0$, то $\Sigma_{j}$ - гиперповерхность $\mathscr{F}_{j}^{*}$, образованная точками, для которых функция

$$
S_{j}\left(t_{j}, \lambda_{1}^{j}, \ldots, \lambda_{n_{j}}^{j}\right)=t_{j}^{n_{j}+1}+\lambda_{n_{j}}^{j} t_{j}^{n_{j}-1}+\cdots+\lambda_{2}^{j} t_{j}+\lambda_{1}^{j}
$$

как многочлен от $t_{j}$ имеет кратный вещественный корень;

- если $m_{j}>0$, то $\Sigma_{j}$ - обвединение следующих гиперповерхностей: 
а) гиперповерхности $\mathscr{F}_{j}^{*}$, образованной точками, для которых функuия

$$
S_{j}\left(t_{j}, \lambda_{m_{j}}^{j}, \ldots, \lambda_{n_{j}}^{j}\right)=t_{j}^{n_{j}-m_{j}+1}+\sum_{i=m_{j}}^{n_{j}} \lambda_{i}^{j} t_{j}^{i-m_{j}}
$$

как многочлен от $t_{j}$ имеет кратный вещественный корень (гиперповерхность $\mathscr{F}_{j}^{*}$ не пуста только при $n_{j}>m_{j}$ );

b) $m_{j}$ гладких гиперповерхностей $H_{i}^{j}, i=1, \ldots, m_{j}$, заданньх уравнениями

$$
\begin{aligned}
H_{i}^{j}: & \left.S_{j}\right|_{t_{j}=-\left(\lambda_{i}^{j}+\cdots+\lambda_{m_{j}-1}^{j}=0\right)}=0, \quad i<m_{j} ; \\
H_{m_{j}}^{j}: & \left.S_{j}\right|_{t_{j}=0}=0
\end{aligned}
$$

(этот набор гиперповерхностей не пуст только при $n_{j}>m_{j}-1$ ); с) $m_{j}\left(m_{j}-1\right) / 2$ әиперплоскостей $\Pi_{i, k}^{j}$, заданных уравнениями

$$
t_{i}^{j}+\cdots+t_{k-1}^{j}=0, \quad 1 \leqslant i<k \leqslant m_{j}
$$

( этот набор гиперповерхностей не пуст только при $m_{j}>1$ ).

3) Ростки гиперповерхностей $\mathscr{F}_{j}^{*}, H_{i}^{j}, \Pi_{i, k}^{j}$ в нуле являются полными прообразами ростков множеств

$$
\begin{aligned}
& {\overline{\left(\mathscr{A}^{j}+A_{1}\right)}}_{\mathscr{F}_{j}}, \quad{\overline{\left(\mathscr{A}^{j}-A_{\mu_{(i, j)}}+A_{\left.\mu_{(i, j)}+1\right)}\right.}}_{\mathscr{F}_{j}}, \\
& {\overline{\left(\mathscr{A}^{j}-A_{\mu_{(i, j)}}-A_{\mu_{(k, j)}}+A_{\mu_{(i, j)}}+\mu_{(k, j)}+1\right)}}_{\mathscr{F}_{j}}
\end{aligned}
$$

в точке $x$ соответственно относительно ростка отобрахсения $\varphi_{\mathscr{A}}$ в точке $\xi_{m}$. Здесь $\mathscr{A}^{j}=\sum_{l \in I_{j}} A_{\mu_{(l, j)}}$, а индекс $(l, j)$ является l-м числом в набоpe $I_{j}$.

4) Росток обгединения $\mathscr{F}^{*}=\bigcup_{j=1}^{p} \mathscr{F}_{j}^{*}$ в нуле является ростком фронта $[\mathscr{A}](\mathscr{F}) \subset \Phi_{\mathscr{A}}$ в точке $\xi_{m}$. Этот росток является полним прообразом ростка множсества $\overline{\left(\mathscr{A}+A_{1}\right)}$ F्ञ относительно ростка отображсения $\varphi_{\mathscr{A}}$ в точке $\xi_{m}$.

ДокАЗАТЕЛьСтво. Согласно [1], [14] существуют локальные координаты

$$
\begin{aligned}
\left(q_{0}^{1}, \ldots, q_{\nu_{1}-1}^{1}, \ldots, q_{0}^{p}, \ldots, q_{\nu_{p}-1}^{p}, z_{1}, \ldots, z_{n-\operatorname{codim} X}\right) \\
\in \mathbb{R}^{n}=\mathbb{R}^{\nu_{1}} \times \cdots \times \mathbb{R}^{\nu_{p}} \times \mathbb{R}^{n-\operatorname{codim} X}
\end{aligned}
$$

на многообразии $V$ такие, что для любого $j=1, \ldots, p$ компонента $\mathscr{F}_{j}$ является ростком в нуле цилиндра, у которого

1) направляющей служит гиперповерхность $\Delta^{j} \subset \mathbb{R}^{\nu_{j}}=\left(q_{0}^{j}, \ldots, q_{\nu_{j}-1}^{j}\right)$, образованная точками, для которых многочлен

$$
t_{j}^{\nu_{j}+1}+q_{\nu_{j}-1}^{j} t_{j}^{\nu_{j}-1}+\cdots+q_{1}^{j} t_{j}+q_{0}^{j}
$$

от $t_{j}$ имеет кратный вешественный корень;

2) образующие коллинеарны плоскости $q_{0}^{j}=\cdots=q_{\nu_{j}-1}^{j}=0$. 
Отсюда следует, что существуют разложение $\mathscr{A}=\mathscr{A}^{1}+\cdots+\mathscr{A}^{p}$ и локальные координаты

$$
\left(\lambda_{1}^{1}, \ldots, \lambda_{n_{1}}^{1}, \ldots, \lambda_{1}^{p}, \ldots, \lambda_{n_{p}}^{p}, z_{1}, \ldots, z_{n-\operatorname{codim} X}\right), \quad n_{j}=\nu_{j}-\operatorname{codim} \mathscr{A}^{j}
$$

на многообразии $\Phi_{\mathscr{A}}$ такие, что в этих координатах росток $\left(\operatorname{St}_{1}\left(\Phi_{\mathscr{A}}\right), \xi_{m}\right)$ является ростком в нуле объединения $\bigcup_{j=1}^{p} \Sigma_{j}$ гиперповерхностей $\Sigma_{j}$, которые определяются следуюшим образом:

- если $\mathscr{A}^{j}=\emptyset$, то $\left(\lambda_{1}^{j}, \ldots, \lambda_{n_{j}}^{j}\right)=\left(q_{0}^{j}, \ldots, q_{\nu_{j}-1}^{j}\right)$ и $\Sigma_{j}$ является цилиндром, у которого направляющей служит фронт $\Delta^{j} \subset \mathbb{R}^{\nu_{j}}=\left(\lambda_{1}^{j}, \ldots, \lambda_{n_{j}}^{j}\right)$, а образующие коллинеарны плоскости $\lambda_{1}^{j}=\cdots=\lambda_{n_{j}}^{j}=0$;

- если $\mathscr{A}^{j} \neq \emptyset$, то $\Sigma_{j}$ является цилиндром, у которого направляющей служит остов коразмерности 1 канонической стратификации характеристического многообразия особенности $\mathscr{A}^{j}$ фронта $\Delta^{j} \subset \mathbb{R}^{\nu_{j}}=\left(q_{0}^{j}, \ldots, q_{\nu_{j}-1}^{j}\right)$, а образующие коллинеарны плоскости $\lambda_{1}^{j}=\cdots=\lambda_{n_{j}}^{j}=0$.

Теперь предложение 6.4 следует из предложения 6.2 .

Теорема 4.7 является следствием предложения 6.4 .

\section{§7. Доказательство теоремы 3.5}

Пусть $\mathscr{F}$ - фронт устойчивого лежандрова отображения $f: L \rightarrow V$ коранга 1 в гладком $n$-мерном многообразии $V$. Рассмотрим произвольную правильную связную компоненту $U$ дополнения $V \backslash \mathscr{F}$. Через $Г$ обозначим край этой компоненты.

Зафиксируем произвольное натуральное $\mu$ и рассмотрим характеристическое многообразие $\Phi_{A_{\mu}} \subseteq L$ особенности $A_{\mu}$ фронта $\mathscr{F}$, характеристическое отображение $\varphi_{A_{\mu}}: \Phi_{A_{\mu}} \rightarrow V$, фронт $\left[A_{\mu}\right](\mathscr{F}) \subset \Phi_{A_{\mu}}$ и замыкание ${\overline{\left(A_{\mu}\right)}}_{\Gamma}$ многообразия $\left(A_{\mu}\right)_{\Gamma}$ особенностей $A_{\mu}$ гиперповерхности Г. Через $\Omega$ обозначим полньй прообраз множества ${\overline{\left(A_{\mu}\right)}}_{\Gamma}$ при отображении $\varphi_{A_{\mu}}$.

ПРЕДЛОЖЕНИЕ 7.1. Пусть фронт $\mathscr{F}$ имеет особенность $A_{\nu}$ в точке $x \in$ ${\overline{\left(A_{\mu}\right)}}_{\Gamma}$, а компонента $U$ является гиперболической (обобщенно-эллиптической) в этой точке. Тогда:

1) если $\nu=\mu$ или $\mu+1$, то росток $(\Omega, \xi)$ множсества $\Omega$ в точке $\xi=$ $\varphi_{A_{\mu}}^{-1}(x)$ является ростком многообразия $\Phi_{A_{\mu}}$;

2) если $\nu>\mu+1$, то росток $(\Omega, \xi)$ является ростком замыкания связной компоненты дополнения $\Phi_{A_{\mu}} \backslash\left[A_{\mu}\right](\mathscr{F})$; әта компонента является гиперболической (обобщенно-эллиптической соответственно) в точке $\xi$.

ДокАЗАТЕЛЬство. Как и при доказательстве предложения 6.2, можно считать, что росток отображения $f$ в точке $\xi$ задается формулой

$$
(f, \xi):\left(\mathbb{R}^{n-1}, 0\right) \rightarrow\left(\mathbb{R}^{n}, 0\right), \quad(t, \bar{q}) \mapsto\left(-\frac{\partial}{\partial t} S(t, \bar{q}), \bar{q}, S(t, \bar{q})-t \frac{\partial}{\partial t} S(t, \bar{q})\right)
$$


где

$$
\bar{q}=\left(q_{2}, \ldots, q_{n-1}\right), \quad S(t, \bar{q})=t^{\nu+1}+q_{\nu-1} t^{\nu-1}+\cdots+q_{2} t^{2}
$$

Тогда росток многообразия $\Phi_{A_{\mu}}$ в этой точке задается уравнениями

$$
\frac{\partial^{2} S}{\partial t^{2}}=\frac{\partial^{3} S}{\partial t^{3}}=\cdots=\frac{\partial^{\mu} S}{\partial t^{\mu}}=0
$$

Росток $(\Omega, \xi)$ - росток в нуле замыкания множества точек $(t, \bar{q}) \in \Phi_{A_{\mu}} \backslash \Phi_{A_{\mu+1}}$, для которых функция

$$
F(u, t, \bar{q})=S(u, \bar{q})-S(t, \bar{q})-(u-t) \frac{\partial}{\partial t} S(t, \bar{q})
$$

как многочлен от $u$ имеет максимально возможное число простых вещественных корней (минимально возможное число вешественных корней с учетом их кратностей, если компонента $U$ обобщенно-эллиптическая в точке $x$ ). Согласно лемме 5.5 последнее равносильно тому, что функция

$$
\sum_{i=\mu}^{\nu} \frac{1}{(i+1) !} \frac{\partial^{i+1} S(t, \bar{q})}{\partial t^{i+1}}(u-t)^{i-\mu}
$$

как многочлен от $u-t$ имеет $\nu-\mu$ простых вещественных корней (соответственно не имеет вешественных корней, если $\nu-\mu$ четное, и имеет один простой вещественный корень, если $\nu-\mu$ нечетное).

Предложение 7.1 следует теперь из предложения 6.1.

ПРЕДЛОЖЕНИЕ 7.2. Множество $\Omega$ является многообразием $с$ краем. Каждая связная компонента этого многообразия представляет собой замыкание правильной связной компоненты дополнения $\Phi_{A_{\mu}} \backslash\left[A_{\mu}\right](\mathscr{F})$.

ДокаЗАтельство. Пусть $\xi \in \Omega$ и фронт $\mathscr{F}$ имеет особенность $X=A_{\nu_{1}}+$ $\cdots+A_{\nu_{p}}$ в точке $x=\varphi_{A_{\mu}}(\xi)$. Рассмотрим неприводимые компоненты $\mathscr{F}_{1}, \ldots, \mathscr{F}_{p}$ ростка $(\mathscr{F}, x)$ и предположим, что эти компоненты имеют в точке $x$ особенности $A_{\nu_{1}}, \ldots, A_{\nu_{p}}$ соответственно. Как и при доказательстве предложения 6.4 , выберем локальные координаты

$$
\begin{aligned}
\left(q_{0}^{1}, \ldots, q_{\nu_{1}-1}^{1}, \ldots, q_{0}^{p}, \ldots, q_{\nu_{p}-1}^{p}, z_{1}, \ldots, z_{n-\operatorname{codim} X}\right) \\
\in \mathbb{R}^{n}=\mathbb{R}^{\nu_{1}} \times \cdots \times \mathbb{R}^{\nu_{p}} \times \mathbb{R}^{n-\operatorname{codim} X}
\end{aligned}
$$

на многообразии $V$ такие, что для любого $j=1, \ldots, p$ компонента $\mathscr{F} j$ является ростком в нуле гиперповерхности $\Delta_{\nu_{j}}(n) \subset \mathbb{R}^{n}$, образованной точками, для которых многочлен

$$
P_{j}\left(t_{j}\right)=t_{j}^{\nu_{j}+1}+q_{\nu_{j}-1}^{j} t_{j}^{\nu_{j}-1}+\cdots+q_{1}^{j} t_{j}+q_{0}^{j}
$$

имеет кратньй вещественньй корень.

В этих координатах росток замькания $\bar{U}$ области $U$ в точке $x$ является ростком в нуле пересечения $\bigcap_{j=1}^{p} \bar{U}_{j}$, где $\bar{U}_{j}$ - замькание правильной связной компоненты $U_{j}$ дополнения к гиперповерхности $\Delta_{\nu_{j}}(n)$. Каждая из этих компонент либо гиперболическая, либо обобщенно-эллиптическая независимо друг от друга. 
Пусть, для определенности, $\mathscr{F}_{1}$ - фронт ростка многообразия $L$ в точке $\xi$. Тогда набор

$$
\left(t_{1}, q_{\mu+1}^{1}, \ldots, q_{\nu_{1}-1}^{1}, q_{0}^{2}, \ldots, q_{\nu_{2}-1}^{2}, \ldots, q_{0}^{p}, \ldots, q_{\nu_{p}-1}^{p}, z_{1}, \ldots, z_{n-\operatorname{codim} X}\right) \in \mathbb{R}^{n-\mu}
$$

определяет локальные координаты на многообразии $\Phi_{A_{\mu}}$ в окрестности точки $\xi$. В этих координатах росток $(\Omega, \xi)$ является ростком в нуле пересечения $\bigcap_{j=1}^{p} \Omega_{j}$ подмножеств $\Omega_{j}$ в $\mathbb{R}^{n-\mu}$, которые определяются следуюшим образом:

1) $\Omega_{1}$-полньй прообраз замыкания $\overline{\left(A_{\mu}\right)} \Gamma_{1}$ многообразия особенностей $A_{\mu}$ на крае $\Gamma_{1}$ компоненты $U_{1}$ относительно ростка отображения $\varphi_{A_{\mu}}$ в точке $\xi$;

2) если $j>1$, то $\Omega_{j}$ - замыкание правильной связной компоненты дополнения к гиперповерхности в $\mathbb{R}^{n-\mu}$, образованной точками, для которых многочлен $P_{j}$ имеет кратный вешественный корень; эта компонента гиперболическая (обобшенно-эллиптическая), если компонента $U_{j}$ гиперболическая (обобшенно-эллиптическая соответственно).

Предложение 7.2 следует теперь из предложения 7.1.

Теорема 3.5 является следствием предложений 7.2 и 6.4 .

\section{§8. Специальные фронты в $\mathbb{R}^{n}$}

В этом параграфе построены некоторые специальные фронты в евклидовом пространстве $\mathbb{R}^{n}$. Они нужны для доказательства теоремы 1.10 .

ПРЕДЛОЖЕНИЕ 8.1. Пусть $\mathscr{F ~ - ~ к о м п а к т н ъ и ̆ ~ у с т о и ̆ ч и в ы и ̆ ~ ф р о н т ~ к о р а н г а ~} 1$ в $\mathbb{R}^{n}$, где $n>2$. Предположим, что дополнение $\kappa \mathscr{F}$ имеет гиперболическую (обобщенно-эллиптическую, әллиптическую) ограниченную связную компоненту $U$, край $\Gamma$ которой состочт из точек нескольких неприводимых ком-

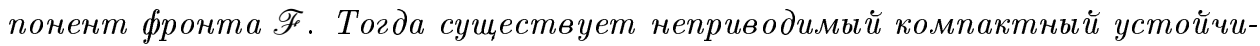
вый фронт $\widetilde{\mathscr{F}}$ коранга 1 в $\mathbb{R}^{n}$ такой, что дополнение $\kappa \widetilde{\mathscr{F}}$ имеет гиперболическую (обобщенно-эллиптическую, әллиптическую соответственно) ограниченную связную компоненту, край $\widetilde{\Gamma}$ которой удовлетворяет следующему условию: многообразие $\mathscr{A}_{\widetilde{\Gamma}}$ особенностей $\mathscr{A}$ гиперповерхности $\widetilde{\Gamma}$ совпадает с многообразием $\mathscr{A}_{\Gamma}$ особенностей $\mathscr{A}$ гиперповерхности Г для любого $\mathscr{A} \neq A_{1}$.

ДоКАЗАТЕЛЬСТво. Предположим, что гиперповерхность Г состоит из точек $k$ неприводимых компонент $\mathscr{F}_{1}, \ldots, \mathscr{F}_{k}$ фронта $\mathscr{F}$, где $k \geqslant 2$. Тогда найдутся $C^{\infty}$-вложенные кривые $\gamma_{i}:[a, b] \subset \mathbb{R} \rightarrow \mathbb{R}^{n}, i=1, \ldots, k-1$, такие, что:

1) каждая кривая $\gamma_{i}$ пересекает фронт $\mathscr{F}$ ровно в двух гладких точках $P_{i}^{1}, P_{i}^{2}$, причем трансверсально;

2) $P_{i}^{1}=\gamma_{i}\left(t_{1}\right) \in \mathscr{F}_{i}$, a $P_{i}^{2}=\gamma_{i}\left(t_{2}\right) \in \mathscr{F}_{i+1}$, где $a<t_{1}<t_{2}<b$ и $\gamma_{i}\left(t_{1}, t_{2}\right) \subset U$;

3) кривые $\gamma_{1}, \ldots, \gamma_{k-1}$ попарно не пересекаются.

Возьмем произвольное $\varepsilon>0$ и рассмотрим $\varepsilon$-окрестности $O_{i}$ кривых $\gamma_{i}$ в $\mathbb{R}^{n}$. Если $\varepsilon$ достаточно мало, то замыкания $\bar{O}_{1}, \ldots, \bar{O}_{k-1}$ этих окрестностей попарно не пересекаются; каждое пересечение $\bar{O}_{i} \cap \mathscr{F}$ состоит только из гладких точек фронта $\mathscr{F}$ и имеет ровно две связные компоненты $D_{i}^{1} \ni P_{i}^{1}, D_{i}^{2} \ni P_{i}^{2}$; подмножества $D_{i}^{1}, D_{i}^{2} \subset \mathbb{R}^{n}$ являются гладко вложенными замкнутыми $(n-1)$-мерными дисками с гладкими краями $\partial D_{i}^{1}, \partial D_{i}^{2}$ (диффеоморфными $(n-2)$-мерной сфере $S^{n-2}$ ); 
каждое пересечение $C_{i}=\partial O_{i} \cap \bar{U}$, где $\partial O_{i}$ - граница окрестности $O_{i}$, а $\bar{U}$ - замыкание компоненты $U$, является гладко вложенным цилиндром $S^{n-2} \times\left[t_{1}, t_{2}\right]$.

Следовательно, искомьй фронт $\widetilde{\mathscr{F}}$ можно получить из множества

$$
\left(\left(\bigcup_{i=1}^{k} \mathscr{F}_{i}\right) \backslash \bigcup_{i=1}^{k-1}\left(D_{i}^{1} \cup D_{i}^{2}\right)\right) \cup\left(\bigcup_{i=1}^{k-1} C_{i}\right)
$$

путем стандартного сглаживания (использующего разбиение единицы) в малых окрестностях сфер $\partial D_{i}^{1}, \partial D_{i}^{2}, i=1, \ldots, k-1$. Предложение 8.1 доказано.

ЗАМЕчАнИЕ 8.2. Фронт $\widetilde{\mathscr{F}}$ является связной суммой фронтов $\mathscr{F} 1, \ldots, \mathscr{F} k$ (см. [15]). Это суммирование проведено так, чтобы дополнение к фронту $\widetilde{F}$ имело связную компоненту с необходимыми нам свойствами.

ПрЕДЛОЖЕНИЕ 8.3. Пусть $\mathscr{A}=A_{\mu_{1}}+\cdots+A_{\mu_{m}}$, әде $\mu_{1}+\cdots+\mu_{m}=n>1$. Тогда существуют неприводимъе компактнье устойчивье фронты $\Sigma^{-}(\mathscr{A})$ и $\Sigma^{+}(\mathscr{A})$ коранга 1 в $\mathbb{R}^{n}$ такие, что:

1) дополнение $\kappa \Sigma^{-}(\mathscr{A})\left(\Sigma^{+}(\mathscr{A})\right)$ имеет гиперболическую (обобщенно-әллиптическую) ограниченную связную компоненту $U^{-}\left(U^{+}\right.$соответственно); если все кратности $\mu_{1}, \ldots, \mu_{m}$ нечетные, то компоненma $U^{+}$әллиптическая;

2) край любой из компонент $U^{-}, U^{+}$имеет ровно две особенности $\mathscr{A} и$ не имеет других изолированных особенностей.

ДоКАЗАТЕЛЬСТво. При $n=2$ утверждение очевидно. Подходящими фронтами являются, например, следуюшие кривые на плоскости $x, y$ :

$$
\begin{aligned}
\Sigma^{-}\left(2 A_{1}\right)=\Sigma^{+}\left(2 A_{1}\right): & x=\sin t, & y & =\cos 3 t ; \\
\Sigma^{-}\left(A_{2}\right): & x=\sin t, & y & =\cos ^{3} t ; \\
\Sigma^{+}\left(A_{2}\right): & x=\frac{\sin t}{\sin ^{2} t+\cos ^{6} t}, & y & =\frac{\cos ^{3} t}{\sin ^{2} t+\cos ^{6} t} .
\end{aligned}
$$

Поэтому далее до конца доказательства этого утверждения мы будем предполагать, что $n>2$.

Пусть $\Sigma_{i}-$ фронт в

$$
\mathbb{R}^{n+1}=\left\{\left(\lambda_{0}^{1}, \lambda_{1}^{1}, \ldots, \lambda_{\mu_{1}-1}^{1}, \ldots, \lambda_{0}^{m}, \lambda_{1}^{m}, \ldots, \lambda_{\mu_{m}-1}^{m}, \lambda_{*}\right)\right\},
$$

образованный точками, для которых многочлен

$$
t^{\mu_{i}+1}+\lambda_{\mu_{i}-1}^{i} t^{\mu_{i}-1}+\cdots+\lambda_{1}^{i} t+\lambda_{0}^{i}
$$

от $t$ имеет кратный вещественный корень. Через $U_{i}^{-}\left(U_{i}^{+}\right)$обозначим гиперболическую (обобщенно-эллиптическую соответственно) правильную связную компоненту дополнения $\mathbb{R}^{n+1} \backslash \Sigma_{i}$.

Легко видеть, что гиперповерхность $\Sigma=\bigcup_{i=1}^{m} \Sigma_{i}$ является устойчивым фронтом коранга 1 в $\mathbb{R}^{n+1}$. Множества $U^{-}=\bigcap_{i=1}^{m} U_{i}^{-}$и $U^{+}=\bigcap_{i=1}^{m} U_{i}^{+}$являются 
правильными связными компонентами дополнения $\mathbb{R}^{n+1} \backslash \Sigma$ (гиперболической и обобщенно-эллиптической соответственно).

Рассмотрим стандартную сферу $S^{n}$ в $\mathbb{R}^{n+1}$ с центром в нуле. Пересечение $\Sigma \cap S^{n}$ является компактным устойчивым фронтом коранга 1 на $S^{n}$. Этот фронт имеет ровно две изолированные особенности, и обе они являются особенностями $\mathscr{A}$.

Пересечения $U^{-} \cap S^{n}$ и $U^{+} \cap S^{n}$ являются гиперболической и обобщенно-эллиптической соответственно связными компонентами дополнения $S^{n} \backslash\left(\Sigma \cap S^{n}\right)$. Край любой из них содержит обе особенности $\mathscr{A}$ фронта $\Sigma \cap S^{n}$. Компонента $U^{+} \cap S^{n}$ является эллиптической, если все числа $\mu_{1}, \ldots, \mu_{m}$ нечетные.

Зафиксируем произвольную точку $P \in S^{n}$ и рассмотрим гиперплоскость $\pi$ в $\mathbb{R}^{n+1}$, касающуюся сферы $S^{n}$ в точке, диаметрально противоположной $P$. Через $\sigma_{P}$ обозначим стереографическую проекцию гиперплоскости $\pi$ на $S^{n}$ с полю$\operatorname{com} P$.

Прообраз фронта $\Sigma \cap S^{n}$ и прообраз множества $U^{-} \cap S^{n}$ относительно проекции $\sigma_{P}$, где $P \in S^{n} \backslash\left(\Sigma \cup U^{-}\right)$(прообраз фронта $\Sigma \cap S^{n}$ и прообраз множества $U^{+} \cap S^{n}$ относительно проекции $\sigma_{P}$, где $\left.P \in S^{n} \backslash\left(\Sigma \cup U^{+}\right)\right)$являются фронтом в $\mathbb{R}^{n}$ и гиперболической (обобшенно-эллиптической соответственно) ограниченной связной компонентой его дополнения, которые обладают всеми нужными свойствами, кроме неприводимости фронта. Предложение 8.3 следует теперь из предложения 8.1 .

ПреДЛОЖЕНИЕ 8.4. Пусть $\mathscr{A}=A_{\mu_{1}}+\cdots+A_{\mu_{m}}$, әде $1<c=\mu_{1}+\cdots+\mu_{m} \leqslant n$. Тогда существуют неприводимые компактные устойчивые фронты $\Sigma_{n}^{-}(\mathscr{A}) u$ $\Sigma_{n}^{+}(\mathscr{A})$ коранга 1 в $\mathbb{R}^{n}$ такие, что:

1) дополнение $\kappa \Sigma_{n}^{-}(\mathscr{A})\left(\Sigma_{n}^{+}(\mathscr{A})\right)$ имеет гиперболическую (обобщенно-эллиптическую) ограниченную связную компоненту $U^{-}\left(U^{+}\right.$соответственно); если все кратности $\mu_{1}, \ldots, \mu_{m}$ нечетные, то компонента $U^{+}$әллиптическая;

2) край любой из компонент $U^{-}, U^{+}$не имеет особенностей коразмерности выше $c$, а все его особенности коразмерности с являются особенностями $\mathscr{A}$;

3) многообразие, образованное особенностями $\mathscr{A}$ края любой из компонент $U^{-}, U^{+}$, диффеоморфно несвязному обвединению двух сфер размерности $n-c$.

ДокАЗАТЕЛЬСтво. Если $c<n$, то искомые фронты диффеоморфны фронтам $\Sigma^{-}(\mathscr{A}) \times S^{n-c}$ и $\Sigma^{+}(\mathscr{A}) \times S^{n-c}$ в прямом произведении $\mathbb{R}^{c} \times S^{n-c}$, где $\Sigma^{-}(\mathscr{A})$ и $\Sigma^{+}(\mathscr{A})$ - неприводимые компактные устойчивые фронты коранга 1 в $\mathbb{R}^{c}$, обладающие свойствами 1), 2) из предложения 8.3, а $S^{n-c}-$ сфера размерности $n-c$. Если же $c=n$, то предложение 8.4 вытекает из предложения 8.3 при помощи следующего утверждения.

ПрЕДЛОЖЕНИЕ 8.5. Пусть $\mathscr{F}_{1}, \ldots, \mathscr{F}_{k}$ - неприводимые компактные устойчивье фронты коранга 1 в $\mathbb{R}^{n}$, әде $n>1$. Предположсим, что эти фронты располохсены в $k$ попарно не пересекающихся открытых шарах в $\mathbb{R}^{n}$. Предположим также, что для каждого $i=1, \ldots, k$ дополнение $к$ фронту $\mathscr{F}_{i}$ 
имеет гиперболическую (обобщенно-эллиптическую, әллиптическую) ограниченную связную компоненту $U_{i}$ с краем $\Gamma_{i}$. Тогда существует неприводимый

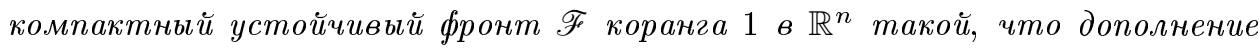
$\kappa$ F имеет гиперболическую (обобщенно-эллиптическую, әллиптическую соответственно) ограниченную связную компоненту, край Г которой обладает следующими свойствами:

1) многообразие $\mathscr{A}_{\Gamma}$ особенностей $\mathscr{A}$ гиперповерхности Г совпадает с (несвязным) обгединением многообразий $\mathscr{A}_{\Gamma_{1}}, \ldots, \mathscr{A}_{\Gamma_{k}}$ особенностей $\mathscr{A}$ гиперповерхностей $\Gamma_{1}, \ldots, \Gamma_{k}$ для любого $\mathscr{A} \neq A_{1}$;

2) эйлерова характеристика многообразия $\left(A_{1}\right)_{\Gamma}$ особенностей $A_{1}$ гиперповерхности Г меньше суммы эйлеровых характеристик многообразий $\left(A_{1}\right)_{\Gamma_{1}}, \ldots,\left(A_{1}\right)_{\Gamma_{k}}$ особенностей $A_{1}$ гиперповерхностей $\Gamma_{1}, \ldots, \Gamma_{k}$ на $k-1$ эйлерову характеристику $(n-1)$-мерной сферь.

ДокАЗАТЕЛЬство. Пусть $\widetilde{\mathscr{F}}=\mathscr{F}_{1} \cup \cdots \cup \mathscr{F}_{k}$ и $k \geqslant 2$. Тогда найдутся $C^{\infty}$-вложенные кривые $\gamma_{i}:[a, b] \rightarrow \mathbb{R}^{n}, i=1, \ldots, k-1$, такие, что:

1) они пересекают фронт $\widetilde{\mathscr{F}}$ только в гладких точках и только трансверсально;

2) каждая кривая $\gamma_{i}$ пересекает только две гиперповерхности из $\Gamma_{1}, \ldots, \Gamma_{k}$, а именно $\Gamma_{i}$ и $\Gamma_{k}$;

3) кривая $\gamma_{i}$ пересекает каждую из гиперповерхностей $\Gamma_{i}, \Gamma_{k}$ ровно в одной точке: $P_{i}^{1}=\gamma_{i}\left(t_{1}\right) \in \Gamma_{i}$ и $P_{i}^{2}=\gamma_{i}\left(t_{2}\right) \in \Gamma_{k}$, где $a<t_{1}<t_{2}<b$;

4) кривые $\gamma_{1}, \ldots, \gamma_{k-1}$ попарно не пересекаются.

Возьмем произвольное $\varepsilon>0$ и рассмотрим $\varepsilon$-окрестности $O_{\varepsilon}\left(\gamma_{i}\right)$ кривых $\gamma_{i}$ в $\mathbb{R}^{n}$. Если $\varepsilon$ достаточно мало, то замыкания $\bar{O}_{\varepsilon}\left(\gamma_{1}\right), \ldots, \bar{O}_{\varepsilon}\left(\gamma_{k-1}\right)$ этих окрестностей попарно не пересекаются; каждое пересечение $\bar{O}_{\varepsilon}\left(\gamma_{i}\right) \cap \widetilde{\mathscr{F}}$ состоит только из гладких точек фронта $\widetilde{\mathscr{F}}$ и имеет $p_{i} \geqslant 2$ связных компонент $D_{i}^{j}(\varepsilon), j=1, \ldots, p_{i}$, где $D_{i}^{1}(\varepsilon) \ni P_{i}^{1}, D_{i}^{2}(\varepsilon) \ni P_{i}^{2} ;$ каждое подмножество $D_{i}^{j}(\varepsilon) \subset \mathbb{R}^{n}$ является гладко вложенньп замкнутьм $(n-1)$-мерным диском с гладким краем $\partial D_{i}^{j}(\varepsilon)$ (диффеоморфным $(n-2)$-мерной сфере $\left.S^{n-2}\right)$; каждая разность $C_{i}(\varepsilon)=\partial O_{\varepsilon}\left(\gamma_{i}\right) \backslash\left(U_{i} \cup U_{k}\right)$, где $\partial O_{\varepsilon}\left(\gamma_{i}\right)$ - граница окрестности $O_{\varepsilon}\left(\gamma_{i}\right)$, является гладко вложенньм цилиндром $S^{n-2} \times\left[t_{1}, t_{2}\right]$.

Зафиксируем любое такое $\varepsilon$ и выберем гладко вложенные замкнутые $(n-1)$-мерные диски $\widetilde{D}_{i}^{j}(\varepsilon), i=1, \ldots, k, j=3, \ldots, p_{i}$, в $\mathbb{R}^{n}$, которые не пересекают гиперповерхности $\Gamma_{1}, \ldots, \Gamma_{k}$ и множества $\bar{O}_{\varepsilon / 2}\left(\gamma_{1}\right), \ldots, \bar{O}_{\varepsilon / 2}\left(\gamma_{k-1}\right)$, но $\partial \widetilde{D}_{i}^{j}(\varepsilon)=\partial D_{i}^{j}(\varepsilon)$ для всех $i, j$. Тогда искомый фронт получается из множества $(\widetilde{\mathscr{F}} \backslash D) \cup C$, где

$$
\begin{aligned}
& D=\bigcup_{i=1}^{k-1}\left(D_{i}^{1}\left(\frac{\varepsilon}{2}\right) \cup D_{i}^{2}\left(\frac{\varepsilon}{2}\right) \cup D_{i}^{3}(\varepsilon) \cup \cdots \cup D_{i}^{p_{i}}(\varepsilon)\right), \\
& C=\bigcup_{i=1}^{k-1}\left(C_{i}\left(\frac{\varepsilon}{2}\right) \cup \widetilde{D}_{i}^{3}(\varepsilon) \cup \cdots \cup \widetilde{D}_{i}^{p_{i}}(\varepsilon)\right),
\end{aligned}
$$

путем стандартного сглаживания (использующего разбиение единицы) в малых окрестностях сфер $\partial D_{i}^{1}(\varepsilon / 2), \partial D_{i}^{2}(\varepsilon / 2), \partial D_{i}^{3}(\varepsilon), \ldots, \partial D_{i}^{p_{i}}(\varepsilon), i=1, \ldots, k-1$, с последуюшей малой деформацией дисков $\widetilde{D}_{i}^{3}(\varepsilon), \ldots, \widetilde{D}_{i}^{p_{i}}(\varepsilon)$ вне этих окрестностей. Предложение 8.5 доказано. 


\section{§9. Доказательство теоремы 1.10}

Пусть $n>1$ и список особенностей $\mathscr{A}^{1}, \ldots, \mathscr{A}^{N}$ сформирован по неубыванию их коразмерностей. В частности, $\mathscr{A}^{1}=A_{1}$ при нечетном $n$ и $\mathscr{A}^{1}=2 A_{1}$ при четном $n$.

Зафиксируем неприводимые компактные устойчивые фронты

$$
\Sigma_{n}^{-}\left(\mathscr{A}^{2}\right), \quad \Sigma_{n}^{+}\left(\mathscr{A}^{2}\right), \quad \ldots, \quad \Sigma_{n}^{-}\left(\mathscr{A}^{N}\right), \quad \Sigma_{n}^{+}\left(\mathscr{A}^{N}\right)
$$

коранга 1 в $\mathbb{R}^{n}$, обладающие свойствами 1)-3) из предложения 8.4 для $\mathscr{A}=\mathscr{A}^{2}, \ldots, \mathscr{A}^{N}$ соответственно. Через $\Sigma_{n}^{-}\left(\mathscr{A}^{1}\right), \Sigma_{n}^{+}\left(\mathscr{A}^{1}\right)$ обозначим аналогичные фронты для $\mathscr{A}=\mathscr{A}^{1}$, если $n$ четное, и $(n-1)$-мерные торы, гладко вложенные в $\mathbb{R}^{n}$, если $n$ нечетное.

Дополнение к любому фронту $\Sigma_{n}^{-}\left(\mathscr{A}^{i}\right)\left(\Sigma_{n}^{+}\left(\mathscr{A}^{i}\right)\right), i=1, \ldots, N$, имеет гиперболическую (обобщенно-эллиптическую соответственно) ограниченную связную компоненту, край $\Gamma_{i}^{-}\left(\Gamma_{i}^{+}\right)$которой не имеет особенностей коразмерности выше $\operatorname{codim} \mathscr{A}^{i}$, а все его особенности коразмерности соdim $\mathscr{A}^{i}$ являются особенностями $\mathscr{A}^{i}$. Многообразие $\left(\mathscr{A}^{i}\right)_{\Gamma_{i}^{-}}\left(\left(\mathscr{A}^{i}\right)_{\Gamma_{i}^{+}}\right)$особенностей $\mathscr{A}^{i}$ гиперповерхности $\Gamma_{i}^{-}$ $\left(\Gamma_{i}^{+}\right)$диффеоморфин несвязному объединению двух сфер размерности $n-\operatorname{codim} \mathscr{A}^{i}$, если $\mathscr{A}^{i} \neq A_{1}$, и является $(n-1)$-мерным тором, если $\mathscr{A}^{i}=A_{1}$. Гиперповерхность $\Gamma_{i}^{+}$имеет особенности только с нечетными кратностями, если все кратности особенности $\mathscr{A}^{i}$ нечетные.

ДоКАЗАТЕЛЬСТво ТЕОРЕмы 1.10 ДЛя множествА $G_{\text {hyp }}$. Пусть $\chi_{i, j}$ - эйлерова характеристика многообразия $\left(\mathscr{A}^{j}\right)_{\Gamma_{i}^{-}}$особенностей $\mathscr{A}^{j}$ гиперповерхности $\Gamma_{i}^{-}$, где $i, j=1, \ldots, N$. Рассмотрим произвольные целые неотрицательные $k_{1}, \ldots, k_{N}$ такие, что $k_{1}^{2}+\cdots+k_{N}^{2} \neq 0$. Для любого $i=1, \ldots, N$ построим $k_{i}$ фронтов $\mathscr{F}_{i, l}, l=1, \ldots, k_{i}$, в $\mathbb{R}^{n}$, диффеоморфных фронту $\Sigma_{n}^{-}\left(\mathscr{A}^{i}\right)$ и таких, что каждый фронт $\mathscr{F}_{i, l}$ расположен в одном из $k_{1}+\cdots+k_{N}$ попарно не пересекающихся открытых шарах в $\mathbb{R}^{n}$.

$\mathrm{K}$ фронтам $\mathscr{F}_{i, l}, i=1, \ldots, N, l=1, \ldots, k_{i}$, применим предложение 8.5. Согласно этому утверждению существует неприводимый компактный устойчивый фронт $\mathscr{F}$ коранга 1 в $\mathbb{R}^{n}$ такой, что:

1) дополнение к $\mathscr{F}$ имеет гиперболическую ограниченную связную компоненту;

2) эйлерова характеристика многообразия $\left(\mathscr{A}^{j}\right)_{\Gamma}$ особенностей $\mathscr{A}^{j}$ края Г этой компоненты равна $\sum_{i=1}^{N} k_{i} \chi_{i, j}$ для любого $j=2, \ldots, N$;

3) эйлерова характеристика многообразия $\left(\mathscr{A}^{1}\right)_{\Gamma}$ особенностей $\mathscr{A}^{1}$ гиперповерхности $\Gamma$ равна $\sum_{i=1}^{N} k_{i}\left(\chi_{i, 1}-\chi\left(S^{n-1}\right)\right)+\chi\left(S^{n-1}\right)$, где $\chi\left(S^{n-1}\right)-$ эйлерова характеристика $(n-1)$-мерной сферы.

Рассмотрим полугруппу в линейном пространстве $\mathbb{R}^{N}$, состоящую из векторов

$$
\left(\sum_{i=1}^{N} k_{i}\left(\chi_{i, 1}-\chi\left(S^{n-1}\right)\right), \sum_{i=1}^{N} k_{i} \chi_{i, 2}, \ldots, \sum_{i=1}^{N} k_{i} \chi_{i, N}\right),
$$

соответствующих всевозможным упорядоченным наборам целых неотрицательных чисел $\left(k_{1}, \ldots, k_{N}\right)$. Эта полугруппа порождена векторами

$$
\left(\chi_{i, 1}-\chi\left(S^{n-1}\right), \chi_{i, 2}, \ldots, \chi_{i, N}\right), \quad i=1, \ldots, N .
$$


Покажем, что они линейно независимы.

Действительно, $(N \times N)$-матрица $M$, строками которой служат эти векторы (в указанном выше порядке), является нижне-треугольной (по построению $\chi_{i, j}=$ 0 при всех $j>i$ ). На ее главной диагонали стоят ненулевые числа:

$$
\chi_{1,1}-\chi\left(S^{n-1}\right)=-2,
$$

если $\mathscr{A}^{1}=A_{1}$, и

$$
\chi_{i, i}=2 \chi\left(S^{n-\operatorname{codim} \mathscr{A}^{i}}\right)=4
$$

для любого $\mathscr{A}^{i} \neq A_{1}$ (напомним, что $\left.\operatorname{codim} \mathscr{A}^{i} \equiv n \bmod 2\right)$.

Таким образом, $\operatorname{det} M \neq 0$. Это доказывает теорему 1.10 для множества $G_{\text {hyp }}$.

ДоКАЗАТЕЛЬСтво ТЕОРемы 1.10 ДЛЯ множества $G_{\text {elp }}$. Рассмотрим список особенностей $\mathscr{A}^{1}, \ldots, \mathscr{A}^{N}$ и выпишем последовательно особенности $\mathscr{A}^{\alpha(1)}, \ldots$, $\mathscr{A}^{\alpha\left(N_{\text {odd }}\right)}$, имеющие только нечетные кратности. Через $\chi_{i, j}$ обозначим эйлерову характеристику многообразия $\left(\mathscr{A}^{\alpha(j)}\right)_{\Gamma_{\alpha(i)}^{+}}$особенностей $\mathscr{A}^{\alpha(j)}$ гиперповерхности $\Gamma_{\alpha(i)}^{+}, i, j=1, \ldots, N_{\text {odd }}$.

Аналогично предыдущему для любых целых неотрицательных $k_{1}, \ldots, k_{N_{\text {odd }}}$ таких, что $k_{1}^{2}+\cdots+k_{N_{\text {odd }}}^{2} \neq 0$, можно построить неприводимьй компактный устойчивый фронт $\mathscr{F}$ коранга 1 в $\mathbb{R}^{n}$ такой, что:

1) дополнение к $\mathscr{F}$ имеет эллиптическую ограниченную связную компоненту;

2) эйлерова характеристика многообразия $\left(\mathscr{A}^{\alpha(j)}\right)_{\Gamma}$ особенностей $\mathscr{A}^{\alpha(j)}$ края Г этой компоненты равна $\sum_{i=1}^{N_{\text {odd }}} k_{i} \chi_{i, j}$ для любого $j=2, \ldots, N_{\text {odd }}$;

3) эйлерова характеристика многообразия $\left(\mathscr{A}^{\alpha(1)}\right)$ г особенностей $\mathscr{A}^{\alpha(1)}$ гиперповерхности $\Gamma$ равна $\sum_{i=1}^{N_{\text {odd }}} k_{i}\left(\chi_{i, 1}-\chi\left(S^{n-1}\right)\right)+\chi\left(S^{n-1}\right)$.

Как и выше, полугруппа в линейном пространстве $\mathbb{R}^{N_{\text {odd }}}$, состояшая из векторов

$$
\left(\sum_{i=1}^{N_{\text {odd }}} k_{i}\left(\chi_{i, 1}-\chi\left(S^{n-1}\right)\right), \sum_{i=1}^{N_{\text {odd }}} k_{i} \chi_{i, 2}, \ldots, \sum_{i=1}^{N_{\text {odd }}} k_{i} \chi_{i, N_{\text {odd }}}\right)
$$

соответствующих всевозможным упорядоченным наборам целых неотрицательных чисел $\left(k_{1}, \ldots, k_{N_{\text {odd }}}\right)$, порождена $N_{\text {odd }}$ линейно независимыми векторами

$$
\left(\chi_{i, 1}-\chi\left(S^{n-1}\right), \chi_{i, 2}, \ldots, \chi_{i, N_{\text {odd }}}\right), \quad i=1, \ldots, N_{\text {odd }} .
$$

Это доказывает теорему 1.10 для множества $G_{\text {elp }}$. 


\section{Список литературы}

1. Арнольд В.И., Варченко А.Н., Гусейн-Заде С. М. Особенности дифференцируемых отображений. Т. 1. М.: Наука, 1982.

2. Васильев B. А. Лагранжевы и лежандровы характеристические классы. М.: МЦНМО, 2000.

3. Казарян $M$. Э. Характеристические классы лагранжевых и лежандровых особенностей // УМН. 1995. Т. 50. №4. С. 45-70.

4. Седьъ В. Д. Разрешение особенностей коранга 1 фронта общего положения // Функц. анализ и его прилож. 2003. Т. 37. № 2. С. 52-64.

5. Седьx В. Д. Соотношения между эйлеровьми характеристиками многообразий особенностей коранга 1 фронта общего положения // Докл. РАН. 2002. Т. 383. №6. С. 735-739.

6. Мазер Джс. Стратифокации и отображения // УМН. 1972. Т. 27. № 5. С. 85-118.

7. Kleiman S. L. Multiple-point formulas I: Iteration // Acta Math. 1981. V. 147. №1-2. P. $13-49$.

8. Marar W. L., Mond D. Multiple-point schemes for corank 1 maps // J. London Math. Soc. (2). 1989. V. 39. №3. P. 553-567.

9. Goryunov V., Mond D. Vanishing cohomology of singularities of mappings // Compositio Math. 1993. V. 89. № 1. P. 45-80.

10. Romero Fuster M. C. Sphere stratifications and the Gauss map // Proc. Roy. Soc. Edinburgh Sect. A. 1983. V. 95. P. 115-136.

11. Арнольд В.И. Критические точки функций на многообразии с краем, простые группы Ли $B_{k}, C_{k}, F_{4}$ и особенности эволют // УМН. 1978. Т. 33. № 5. С. 91-105.

12. Lojasiewicz $S$. Ensembles semi-analitiques. Bure sur Yvette: IHES, 1965.

13. Wall C. T. C. Stratified sets: a survey // Lecture Notes in Math. 1971. V. 192. P. 133-140.

14. Закалюкин В. М. Особенности лагранжевых и лежандровых отображений // Дис. ... канд. физ.-матем. наук. М.: МГУ, 1977.

15. Arnold V. I. Plane curves, their invariants, perestroikas and classifications // Adv. in Sov. Math. 1994. V. 21. P. 33-91.

Российский государственньй университет

Поступила в редакцию

нефти и газа им. И. М. Губкина, г. Москва

07.04 .2003 и 25.02 .2004 